\title{
Probing Interfacial Effects on Ionization Energies: The Surprising Banality of Anion-Water Hydrogen Bonding at the Air/Water Interface
}

\author{
Suranjan K. Paul and John M. Herbert* \\ Department of Chemistry and Biochemistry, The Ohio State University, Columbus, Ohio 43210 USA
}

(Dated: March 23, 2021)

\begin{abstract}
Liquid microjet photoelectron spectroscopy is an increasingly common technique to measure vertical ionization energies (VIEs) of aqueous solutes, although the interpretation of these experiments is subject to questions regarding sensitivity to bulk versus interfacial solvation environments. Here, we compute aqueous-phase VIEs for a set of inorganic anions, some of which partition preferentially at the air/water interface, using a combination of molecular dynamics simulations and electronic structure calculations. The results are in excellent agreement with experiment, regardless of whether the simulation data are restricted to ions at the air/water interface or to those in bulk liquid water. Although the computed VIEs are sensitive to ion-water hydrogen bonding, we find that the short-range solvation structure is sufficiently similar in the bulk and interfacial environments that it proves impossible to discriminate between the two on the basis of the VIE, a conclusion that has important implications for the interpretation of liquid-phase photoelectron spectroscopy. More generally, analysis of the simulation data suggests that partitioning of soft anions at the air/water interface is largely a second (or third) solvation shell effect, arising from disruption of water-water hydrogen bonds and not from significant changes in first-shell anion-water hydrogen bonding.
\end{abstract}

\section{Introduction}

Ion hydration and "specific ion" effects, ${ }^{1,2}$ in the sense of Hofmeister's ranking of the lyotropic effects of aqueous ions ${ }^{2-6}$ play a significant role in the chemistry of the air/ water interface. ${ }^{1,7-9}$ This is an importance interface for atmospheric chemistry, ${ }^{10-13}$ where for example interfacial $\mathrm{Cl}^{-}$and $\mathrm{Br}^{-}$are relevant to the chemistry of seawater aerosols, ${ }^{14-17}$ while $\mathrm{NO}_{3}^{-}$plays a role in atmospheric $\mathrm{NO}_{x}$ chemistry. ${ }^{13,18}$ Brine rejection at the seawater/ice interface has profound consequences for vertical circulation in Arctic and Antarctic waters. ${ }^{19,20}$

It is well established that certain ions partition preferentially at the liquid/vapor interface,,$^{1,9,21-29}$ and the effect is reproducible in molecular dynamics (MD) simulations. ${ }^{7,29-39}$ For certain ions, a surface propensity is evident in the potential of mean force corresponding to moving the ion through the air/water interface and into bulk water. ${ }^{31,36,38}$ The trend is illustrated by the halides: $\mathrm{I}^{-}(\mathrm{aq})$ and $\mathrm{Br}^{-}(\mathrm{aq})$ exhibit a clear preference for the interface whereas $\mathrm{Cl}^{-}(\mathrm{aq})$ is something of an intermediate case, and $\mathrm{F}^{-}$(aq) exhibits no surface propensity whatsoever. ${ }^{1,7,31,33,35-37,39}$ Chemical hardness, ${ }^{40-43}$ which is distinct from polarizability, ${ }^{41,44-48}$ is found to correlate with surface propensity and can be used to rationalize why polarizable but multivalent ions such as $\mathrm{SO}_{4}^{2-}, \mathrm{SO}_{3}^{2-}, \mathrm{CO}_{3}^{2-}$, and $\mathrm{PO}_{4}^{3-}$ prefer the bulk environment, whereas soft anions such as $\mathrm{I}^{-}, \mathrm{ClO}_{4}^{-}, \mathrm{SCN}^{-}$, and $\mathrm{NO}_{3}^{-}$exhibit varying degrees of surface propensity. ${ }^{1}$

Surface-selective second harmonic generation (SHG) experiments of soft ions at the air/water interface sug-

*herbert@chemistry.ohio-state.edu gest an enhanced free energy of adsorption, whose magnitude is on the order of a water-water hydrogen bond. ${ }^{24-27}$ Studies of aqueous halides using vibrational sum-frequency generation ${ }^{49}$ (VSFG) report significant distortion of the hydrogen-bonding network at the interface, in the case of the the heavier halides, as indicated by a redshift in the $\mathrm{O}-\mathrm{H}$ stretching band of water. ${ }^{22,23,49-54}$ Shifts in the $\mathrm{O}-\mathrm{H}$ region of the infrared are also reported in the presence of oxyanions $\mathrm{XO}_{3}^{-}(\mathrm{X}=\mathrm{Cl}, \mathrm{Br}, \mathrm{I}){ }^{55}$

The present work considers interfacial anion hydration in the context of solution-phase photoelectron spectroscopy via liquid microjets. ${ }^{56-61}$ Relative to gas-phase photoelectron spectroscopy, interpretation of the microjet results is subject to several interrelated issues regarding probing depth, ${ }^{28,56,59}$ the energy-dependent nature of the electron attenuation length, ${ }^{62-64}$ and the inelastic mean free path of the outgoing photoelectron. ${ }^{62}$ Scattering of the outgoing electron imparts a dependence on the wavelength of the photodetachment laser ${ }^{65-69}$ with changes in peak shapes for near-threshold photoionization. ${ }^{69}$ Additional experimental uncertainty arises due to the presence of streaming potentials at the liquid interface. ${ }^{70-72}$ Some of these issues might be clarified by means of theoretical calculations.

In water, the electron attenuation length ranges from 1-10 $\mathrm{nm}$ depending on the electron's kinetic energy, ${ }^{59,63}$ suggesting that liquid microjet photoelectron spectroscopy is interface sensitive, ${ }^{61}$ albeit with significant contributions from beyond the first monolayer of solvent. ${ }^{73}$ Whereas interfacial effects on the photochemistry $^{74}$ and ultraviolet spectroscopy ${ }^{75,76}$ of small aqueous solutes have been demonstrated, there has been no systematic investigation of whether VIEs themselves are sensitive to the presence of the air/water interface; the only detailed studies concern the rather unique 
case of the hydrated electron. ${ }^{66,77-83}$ It is known that the VIE of liquid water is largely unaffected by dissolved ions, ${ }^{84}$ shifting by $<0.2 \mathrm{eV}$ over an $8 \mathrm{M}$ concentration range, ${ }^{84}$ and the VIE of $\mathrm{I}^{-}(\mathrm{aq})$ is similarly unaffected by concentration. ${ }^{85}$ It is not yet known whether VIEs of aqueous ions are sensitive to the air/water interface or not.

That issue is examined here by computing bulk and interfacial VIEs for 16 common inorganic ions for which experimental data (from liquid microjets) are available, ${ }^{59,86}$ using a combination of MD simulations and electronic structure calculations. Long-range polarization makes a significant contribution to VIEs in water ${ }^{87-95}$ and we incorporate this effect via dielectric continuum boundary conditions around an electronic structure calculation that includes several solvation shells of explicit water molecules. ${ }^{95}$ Excellent agreement with experimental results suggests that our procedure constitutes a useful, general approach for modeling aqueous VIEs.

\section{Computational Methods}

A. Procedure. MD simulations were performed using the polarizable AMOEBA force field, ${ }^{96-98}$ which was developed in part to describe aqueous solvation of inorganic ions. ${ }^{99-101}$ AMOEBA hydration energies compare favorably to quantum chemistry results for ion-water clusters as well as to experimental estimates of single-ion solvation energies. ${ }^{98,99}$ Parameters for $\mathrm{CN}^{-}, \mathrm{Cl}^{-}, \mathrm{Br}^{-}$, $\mathrm{I}^{-}$, and $\mathrm{SO}_{4}^{2-}$ were taken from previous literature, ${ }^{96,101}$ but for the present work we extended the parameterization of AMOEBA to include $\mathrm{NO}_{3}^{-}, \mathrm{NO}_{2}^{-}, \mathrm{ClO}^{-}, \mathrm{ClO}_{2}^{-}$, $\mathrm{ClO}_{3}^{-}, \mathrm{ClO}_{4}^{-}, \mathrm{OCN}^{-}, \mathrm{SCN}^{-}, \mathrm{CO}_{3}^{2-}, \mathrm{SO}_{3}^{2-}$ and $\mathrm{PO}_{4}^{3-}$. (The parameterization follows an established protocol ${ }^{102}$ that is described in Section S1.1 of the Supporting Information.)

Simulations were performed in the $N V T$ ensemble using a $31.3 \AA \times 31.3 \AA \times 31.3 \AA$ periodic simulation cell containing 1,024 water molecules and a single ion, representing solvation in isotropic bulk water. The air/water interface was modeled using a $31.3 \AA \times 31.3 \AA \times 156.7 \AA$ periodic slab. All simulations correspond to a liquid density of $0.997 \mathrm{~g} / \mathrm{cm}^{3}$ and were performed at $T=298 \mathrm{~K}$. Convergence tests (Section S1.2) indicate that radial distribution functions (RDFs) and first-shell coordination numbers are unchanged even in much smaller unit cells, e.g., $18.8 \AA \times 18.8 \AA \times 94.0 \AA$ for the slab simulations. These RDFs are generally in good agreement with published work, where available; see Section S1.2 for a discussion. MD simulations were performed using the TinkeRHP software. ${ }^{103}$

We use a "cluster-continuum" approach to compute VIEs within an electronic structure framework. ${ }^{95,104}$ Clusters of water molecules, encompassing approximately two solvation shells around the ion in question, are extracted at intervals along each MD trajectory. The VIE for the ion-water cluster is then computed using electronic structure theory with dielectric continuum boundary conditions, ${ }^{95}$ based on a Poisson equation solver (PEqS). ${ }^{80,81,105}$ As described in detail elsewhere, ${ }^{81}$ this approach uses a three-dimensional permittivity function $\varepsilon(\mathbf{r})$ to interpolate between the values $\varepsilon=1$ within the atomistic region (described using quantum chemistry), and $\varepsilon=78$ in the continuum region, representing bulk water. A solvent-accessible surface ${ }^{95,106}$ is used to define the boundary across which this interpolation occurs. A permittivity function for the air/water interface can be constructed in similar fashion, ${ }^{80,81,95}$ using the Gibbs dividing surface (GDS) to define the boundary between $\varepsilon=1$ (air) and $\varepsilon=78$ (water). A schematic of this setup is shown in Fig. S16.

Crucially, the continuum boundary conditions used herein contain a "nonequilibrium" correction to the $\mathrm{VIE},{ }^{81,95}$ in which the change in polarization upon sudden (vertical) ionization of the solute is computed using the solvent's optical dielectric constant, $\varepsilon_{\infty}$. For water, whose static dielectric constant $\left(\varepsilon_{0}=78\right)$ is much larger than its optical dielectric constant $\left(\varepsilon_{\infty}=1.8\right)$, the use of conventional (equilibrium) continuum solvation methods in inappropriate for VIE calculations, as these methods implicitly assume immediate reorganization of the solvent upon ionization. ${ }^{95}$ The nonequilibrium correction removes this overpolarization in the final state, and can modify VIEs for aqueous ions by $\gtrsim 1 \mathrm{eV} .{ }^{81}$ Additional details regarding the PEqS calculations can be found in Section S2.3 of the Supporting Information.

Two of the radicals obtained upon ionization (namely, $\mathrm{NO}_{2}$ and $\mathrm{NO}_{3}$ ) exhibit artifactual symmetry breaking at the Hartree-Fock level, which precludes the straightforward use of correlated levels of wave function theory. For that reason, all of the calculations reported here were performed using density functional theory (DFT) with the $\omega$ B97M-V functional. ${ }^{107}$ Symmetry-breaking is often significantly mitigated by DFT, ${ }^{108,109}$ as confirmed in the present cases; see Section S2.2 for a detailed analysis. Basis-set effects are quite small, with double- and triple$\zeta$ VIEs differing by an average of $<0.1 \mathrm{eV}$ (see Table S4). Calculations reported below use the aug-cc-pVTZ basis set for the anion and the $6-31+\mathrm{G}^{*}$ basis set for the explicit water molecules, along with the SG-2 quadrature grid. ${ }^{110}$ (For $\mathrm{Br}^{-}$and $\mathrm{I}^{-}$, we use the aug-cc-pVTZ-PP basis set with the corresponding effective core potential.) Electronic structure calculations were performed using Q-CHem (v. 5.3).111

Although the ions considered here have VIEs that are smaller than that of liquid water (estimated at 11.2$11.7 \mathrm{eV}),{ }^{70,112-114}$ in a finite cluster $\mathrm{X}^{-}\left(\mathrm{H}_{2} \mathrm{O}\right)_{N}$ it can nevertheless be the case that the frontier orbital of $\mathrm{X}^{-}$ is not the highest-occupied molecular orbital (HOMO) of the cluster. As in previous work, ${ }^{81}$ we compute the VIE of $\mathrm{X}^{-}$using an initial guess corresponding to a superposition of fragment densities, with selective ionization of one fragment. The maximum overlap method ${ }^{115,116}$ is then used to relax the orbitals of a non-aufbau determi- 


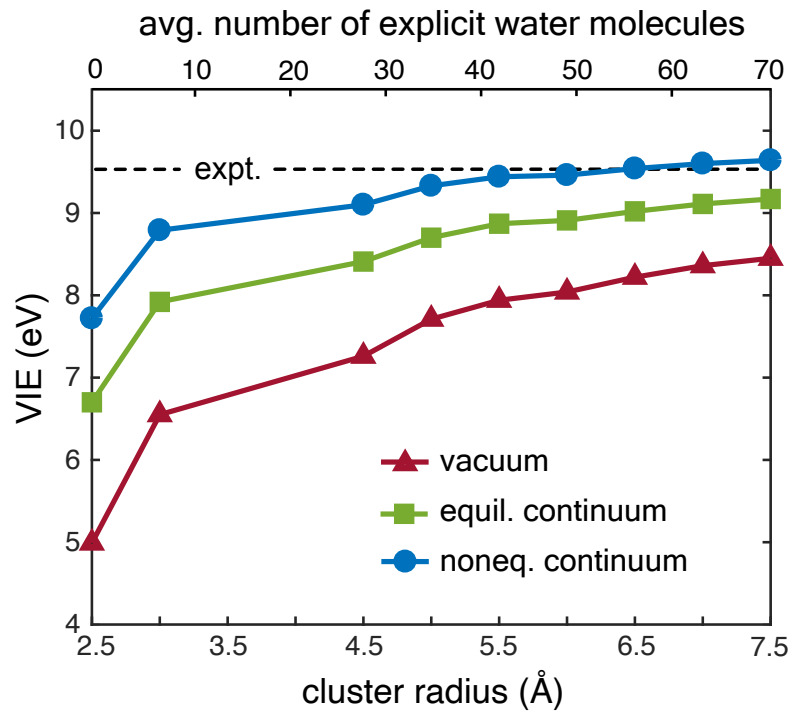

Fig. 1: Vertical ionization energies (VIEs) of increasingly large $\mathrm{NO}_{3}^{-}\left(\mathrm{H}_{2} \mathrm{O}\right)_{N}$ clusters, measured either in terms of the cluster radius (lower horizontal axis) or else the average number of explicit water molecules, $\langle N\rangle$ (upper horizontal axis). All calculations were performed at the $\omega \mathrm{B} 97 \mathrm{M}-\mathrm{V} / 6-31+\mathrm{G}^{*}$ level and each data point represents an average over 25 snapshots extracted from an MD simulation. With equilibrium continuum conditions, both initial and final states are equilibrated using a continuum whose dielectric constant is $\varepsilon_{0}=78$, whereas nonequilibrium boundary conditions polarize the final state using $\varepsilon_{\infty}=1.8$ instead. Vacuum boundary conditions include only the $\mathrm{NO}_{3}^{-}\left(\mathrm{H}_{2} \mathrm{O}\right)_{N}$ cluster but no continuum model The experimental VIE is $9.4 \mathrm{eV}{ }^{86}$

nant corresponding to ionization below the HOMO. More robust convergence algorithms are available for difficult cases, ${ }^{117}$ but we have not found them to be necessary here.

B. Validation. Results from neutron and x-ray diffraction, and from chromatography, establish that even di- and trivalent ions typically disrupt the structure of water only into their second solvation shells, ${ }^{118,119}$ with $\leq 10$ water molecules that are tightly bound to the ion. ${ }^{118}$ Even for exotic ions such as $e^{-}(\mathrm{aq})$, the disruption is limited to two solvation shells. ${ }^{82,83,87}$ Long-range polarization does change dramatically upon ionization, however, leading to very slow convergence of VIEs with respect to the number of explicit water molecules. ${ }^{87-94}$ Consideration of the Born ion model suggests $\sim R^{-1}$ convergence behavior, for an ion-water cluster of radius $R$, and in practice that may mean 500-1000 water molecules to obtain converged results. ${ }^{88-94}$ This problem is not unique to VIEs and is also encountered in $\mathrm{p} K_{\mathrm{a}}$ calculations. ${ }^{104}$

A solution to this conundrum is to use continuum boundary conditions to accelerate convergence with respect to the size of the atomistic solvent cluster. Conver- gence of the VIE for $\mathrm{NO}_{3}^{-}(\mathrm{aq})$ is examined in Fig. 1 using atomistic solutes $\mathrm{NO}_{3}^{-}\left(\mathrm{H}_{2} \mathrm{O}\right)_{N}$ with an increasing number of explicit water molecules, comparing results using both equilibrium and nonequilibrium continuum boundary conditions as well as vacuum boundary conditions. For reasons of computational expedience, these convergence tests were performed not with PEqS but instead using a polarizable continuum model (PCM),${ }^{120-123}$ as convergence properties are similar in both cases. ${ }^{80}$

Convergence to the experimental VIE of $9.4 \mathrm{eV}^{86}$ is obtained using nonequilibrium boundary conditions with approximately two solvation shells of explicit water molecules, corresponding $N \approx 40$ water molecules or a cluster radius $R \approx 6.0 \AA$ around the ion. Similar convergence has been observed in the case of $e^{-}(\mathrm{aq}),{ }^{80}$ which might be considered something of a worst-case scenario due to the delocalized nature of the ion. This is obviously much faster convergence as compared to the hundreds of water molecules suggested above, and indeed it is clear from Fig. 1 that convergence is dramatically slower in the absence of dielectric boundary conditions. Using vacuum boundary conditions, the VIE of $\mathrm{NO}_{3}^{-}\left(\mathrm{H}_{2} \mathrm{O}\right)_{N}$ is $1.0 \mathrm{eV}$ smaller than the experimental value even with $N \approx 70$ explicit water molecules. Boundary conditions based on an equilibrium continuum model (using $\varepsilon_{0}$ only) push that value closer to experiment, but the nonequilibrium ( $\varepsilon_{\infty}$-dependent) correction contributes $0.5 \mathrm{eV}$ in this example and is necessary in order to obtain agreement with experiment. Notably, a "microhydration" approach, including only those water molecules that are directly hydrogen-bonded to the ion (corresponding to $R=3.0 \AA$ in Fig. 1) cannot be recommended with any choice of boundary conditions. Polyvalent ions require a greater number of explicit water molecules to converge the VIE, but additional convergence tests reported in Fig. S17 demonstrate that a cluster extending to $R=7.0 \AA$ is sufficient even for $\mathrm{PO}_{4}^{3-}$ (aq).

\section{Results and Discussion}

A. Comparison to Experiment. By tuning the photon energy and thereby the kinetic energy of the ejected photoelectrons, ${ }^{63}$ liquid microjet photoelectron spectroscopy has been used to measure the concentration profile (versus depth) of ions at the air/water interface. ${ }^{28,124}$ In other measurements, the concentration dependence of the photoelectron yield of aqueous aniline was found to correlate with the surface concentration inferred from surface tension measurements. ${ }^{125}$ If the ionwater hydrogen bonds are different at the interface as compared to those in bulk water, then the VIE might provide a probe of these structural changes. Indeed, comparison of the valence photoelectron spectra of the liquid and vapor phases of water suggests that spectral shifts engendered by hydrogen bonding are different depending on the orbital that is ionized, ${ }^{59}$ and the convergence tests 


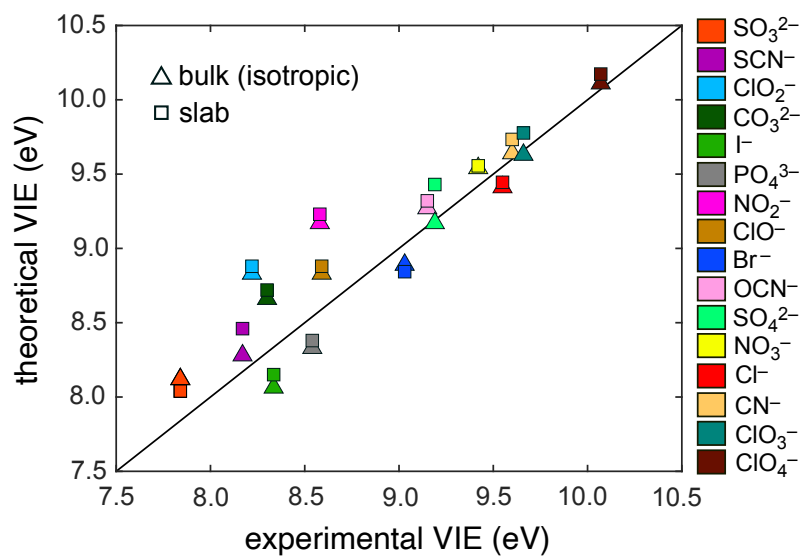

Fig. 2: VIEs for aqueous anions computed from bulk (isotropic) and interfacial (slab) simulations, in comparison to experimental VIEs from Ref. 86. The diagonal line indicates agreement between theory and experiment. Calculations were performed at the $\omega \mathrm{B} 97 \mathrm{M}-\mathrm{V}$ level using nonequilibrium $\mathrm{PEqS}$ boundary conditions, and each data point represents an average over 51 snapshots extracted from the MD simulation. The corresponding numerical data are provided in Table 1.

in Fig. 1 indicate that the VIE is sensitive to short-range ion-water hydrogen bonding. There has been little effort to investigate interfacial effects on VIEs, however, except in the special case of $e^{-}(\mathrm{aq}){ }^{78,80,81}$ and in one preliminary computational study of $\mathrm{F}^{-}(\mathrm{aq}), \mathrm{Cl}^{-}(\mathrm{aq}), \mathrm{Li}^{+}(\mathrm{aq})$, and $\mathrm{Na}^{+}(\mathrm{aq}){ }^{81}$ Of these ions, $e^{-}(\mathrm{aq})$ is rather unique and none of the others is particularly surface active.

Figure 2 shows the correlation between experimental aqueous-phase VIEs for a set of common inorganic ions with theoretical values computed in the present work. (The corresponding numerical data are presented in Table. 1.) Agreement between theory and experiment is very good, with mean absolute errors of $0.2-0.3 \mathrm{eV}$, depending on whether one compares the bulk or the interfacial data set to experiment. Experimental errors are reported to be $0.1-0.3 \mathrm{eV}$ for $\mathrm{I}^{-}(\mathrm{aq})$ and smaller for the other ions. ${ }^{59}$

For calculations in bulk water, continuum boundary conditions can be implemented either by means of a PCM,${ }^{95,121-123}$ which uses a sharp dielectric boundary located at the solvent-accessible surface, or else using a smooth dielectric interface centered around that surface, as in the PEqS approach. ${ }^{80,81,95}$ The variation between VIEs predicted by either approach is $<0.4 \mathrm{eV}$ in all cases (Table 1), which offers some indication of the uncertainty associated with construction of the dielectric boundary. Only the PEqS method is extensible to the air/water interface, and a key result of the present work is that difference between bulk and interfacial VIEs are $<0.1 \mathrm{eV}$, on average.

Direct comparison of experimental and theoretical VIEs is complicated by the fact that the experiments may sample both bulk and interfacial ions, ${ }^{73}$ and the periodic slab calculations definitely sample both environments.
Table 1: VIEs (in eV) for Aqueous Ions Using a ClusterContinuum Approach. ${ }^{a}$

\begin{tabular}{|c|c|c|c|c|c|}
\hline \multirow{3}{*}{ Solute } & \multirow{3}{*}{ Expt. ${ }^{b}$} & \multirow{3}{*}{$\left\langle N_{\mathrm{H}_{2} \mathrm{O}}\right\rangle^{c}$} & \multicolumn{3}{|c|}{ Theory $^{d}$} \\
\hline & & & \multirow{2}{*}{$\begin{array}{l}\text { PCM } \\
\text { (bulk) }\end{array}$} & \multicolumn{2}{|c|}{ PEqS } \\
\hline & & & & bulk & slab \\
\hline $\mathrm{Cl}^{-}$ & 9.60 & 30 & 9.28 & 9.42 & 9.45 \\
\hline $\mathrm{Br}^{-}$ & 9.03 & 45 & 8.50 & 8.89 & 8.84 \\
\hline $\mathrm{I}^{-}$ & $8.34^{e}$ & 43 & 7.90 & 8.07 & 8.14 \\
\hline $\mathrm{CN}^{-}$ & 9.60 & 36 & 9.53 & 9.64 & 9.74 \\
\hline $\mathrm{OCN}^{-}$ & 9.15 & 36 & 9.26 & 9.27 & 9.32 \\
\hline $\mathrm{SCN}^{-}$ & 8.17 & 35 & 8.17 & 8.28 & 8.44 \\
\hline $\mathrm{ClO}^{-}$ & 8.59 & 36 & 8.69 & 8.83 & 8.88 \\
\hline $\mathrm{ClO}_{2}^{-}$ & 8.22 & 36 & 8.57 & 8.84 & 8.91 \\
\hline $\mathrm{ClO}_{3}^{-}$ & 9.66 & 37 & 9.64 & 9.63 & 9.77 \\
\hline $\mathrm{ClO}_{4}^{-}$ & 10.07 & 36 & 10.05 & 10.11 & 10.20 \\
\hline $\mathrm{NO}_{2}^{-}$ & 8.58 & 36 & 9.06 & 9.17 & 9.22 \\
\hline $\mathrm{NO}_{3}^{-}$ & 9.42 & 36 & 9.40 & 9.54 & 9.56 \\
\hline $\mathrm{SO}_{3}^{2-}$ & 7.84 & 61 & 8.01 & 8.12 & 8.03 \\
\hline $\mathrm{SO}_{4}^{2-}$ & 9.19 & 56 & 8.97 & 9.17 & 9.43 \\
\hline $\mathrm{CO}_{3}^{2-}$ & 8.30 & 58 & 8.52 & 8.66 & 8.72 \\
\hline $\mathrm{PO}_{4}^{3-}$ & 8.54 & 59 & 7.88 & 8.33 & 8.41 \\
\hline $\mathrm{MSD}^{f}$ & - & - & -0.05 & 0.10 & 0.17 \\
\hline $\mathrm{MAD}^{g}$ & - & - & 0.23 & 0.21 & 0.26 \\
\hline
\end{tabular}

${ }^{a}$ Simulated VIEs represent averages over 51 snapshots and 500 ps of simulation time. Standard deviations are $\sim 0.3 \mathrm{eV} .{ }^{b}$ From Ref. 86. ${ }^{c}$ Average number of explicit water molecules in the QM region, for the bulk ions. ${ }^{d} \omega \mathrm{B} 97 \mathrm{M}-\mathrm{V}$, with the aug-cc-pVTZ basis set for the ions and $6-31+\mathrm{G}^{*}$ for the explicit water. ${ }^{e}$ Weighted average of spin-orbit states (see text). ${ }^{f}$ Mean signed deviation, theory minus experiment. ${ }^{g}$ Mean absolute deviation.

Values reported in Table 1 represent averages over the entire simulation cell, and certain ions (such as $\mathrm{NO}_{3}^{-}$) move readily between the bulk-like interior of the slab and the air/water interface. As such, it is not obvious which (if any) of the theoretical values should be compared directly to experiment, for a given ion, although this ambiguity is largely mitigated by the close agreement between isotropic and slab VIEs. Agreement with experiment is comparable to the best available periodic DFT calculations, which reported a mean absolute error of $0.1 \mathrm{eV}$ using range-separated hybrid functionals. ${ }^{86}$ The present calculations, however, are free of issues related to the use of periodic electronic structure for ionization or redox processes. ${ }^{126-135}$

Having validated the accuracy of our computational protocol with respect to experimental data, we next turn to the most interesting aspect of the calculations, namely, the similarity between VIEs computed for isotropic versus slab simulations, for the same ion. These differences are $<0.3 \mathrm{eV}$ for each of the ions in our data set, and often much smaller. For $\mathrm{I}^{-}(\mathrm{aq})$ and $\mathrm{SCN}^{-}(\mathrm{aq})$, both 
of which exhibit significant surface propensity, ${ }^{136-142}$ the difference between isotropic and slab VIEs is $0.1 \mathrm{eV}$ and $0.2 \mathrm{eV}$, respectively. Note that for $\mathrm{I}^{-}(\mathrm{aq})$, experimental VIEs are reported corresponding to either of two different spin-orbit states of the ionized species. ${ }^{59}$ Calculations reported here do not include spin-orbit coupling so we use $\left[\operatorname{VIE}\left({ }^{2} P_{1 / 2}\right)+2 \operatorname{VIE}\left({ }^{2} P_{3 / 2}\right)\right] / 3$ as an estimate of the spinorbit-free experimental VIE. ${ }^{143}$

Convergence tests in Fig. S17 suggest that two solvation shells of explicit water molecules, with nonequilibrium Poisson boundary conditions, is sufficient to afford converged VIEs, even for multiply-charged anions. This observation strongly suggests that the electronic structure of these aqueous ions depends only on short-range interactions. The similarity between VIEs computed in isotropic versus slab simulations suggests, in turn, that the short-range ion-water interactions might be very similar in both environments. This hypothesis is investigated in Section 3 C. Before that, we dissect the matter of bulk versus interfacial VIEs in more detail by considering the full ensemble distribution of VIEs, for two different anions that exhibit surface propensities: $\mathrm{I}^{-}(\mathrm{aq})$ and $\mathrm{SCN}^{-}(\mathrm{aq})$.

B. Bulk Versus Interfacial VIEs. We next consider how an ion's position relative to the interface impacts its VIE, focusing specifically on $\mathrm{I}^{-}(\mathrm{aq})$ and $\mathrm{SCN}^{-}(\mathrm{aq})$. Both ions exhibit significant surface propensity and have become canonical examples of surface-active ions. ${ }^{144}$ VSFG experiments demonstrate that both ions have significant effects on the $\mathrm{O}-\mathrm{H}$ stretching band of water. ${ }^{22,23,139,140}$ Halides ions were considered extensively in the very earliest studies of interfacial ion partitioning, ${ }^{1,7}$ but $\mathrm{SCN}^{-}$(aq) at the air/water interface has also been considered in numerous studies. ${ }^{25,29,76,144,145}$ The latter ion has also been considered in the context of Hofmeister "salting-out" effects on proteins. ${ }^{146,147}$

For $\mathrm{I}^{-}(\mathrm{aq})$, our DFT/PEqS calculations afford a VIE of $8.07 \pm 0.28 \mathrm{eV}$ in bulk simulations where there is no interface, as compared to $8.14 \pm 0.31 \mathrm{eV}$ using snapshots extracted from the periodic slab calculations. (Uncertainties represent one standard deviation and characterize inhomogeneous broadening.) Both values are consistent with the experimental VIE, $7.93 \mathrm{eV} .{ }^{86}$ In case of $\mathrm{SCN}^{-}(\mathrm{aq})$, bulk and slab simulations afford VIEs of $8.28 \pm 0.27 \mathrm{eV}$ and $8.44 \pm 0.29 \mathrm{eV}$, respectively, as compared to an experimental VIE of $8.17 \mathrm{eV}{ }^{86}$ For both ions, the difference in the average VIE in the bulk versus the slab simulation is less than the half-width of the VIE distribution obtained upon thermal sampling.

That said, interfacial VIEs reported in Table 1 represent averages over the entire periodic slab, and both $\mathrm{I}^{-}(\mathrm{aq})$ and $\mathrm{SCN}^{-}(\mathrm{aq})$ visit both the interfacial and the bulk-like interior regions of the slab. Supposing that the two regions were to afford very different VIEs, it is conceivable that averaging over the entire slab simulation might mask differences between bulk and interfacial
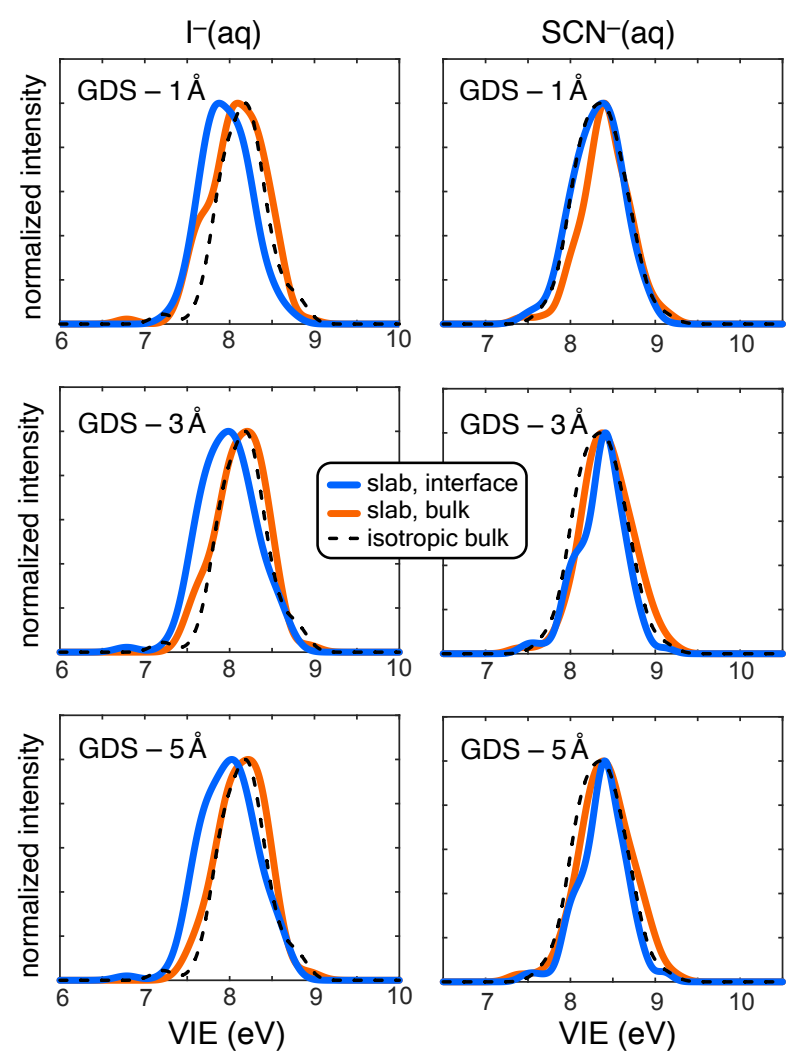

Fig. 3: VIE distributions for $\mathrm{I}^{-}(\mathrm{aq})$ and $\mathrm{SCN}^{-}(\mathrm{aq})$, partitioned into bulk and interfacial contributions according to distance of the ion from the GDS. Also shown are results from an isotropic simulation representing "true bulk". Strong similarities between bulk and interfacial VIE distributions suggest that similar VIEs reported in Table 1 are not simply artifacts of averaging over a slab that contains both interfacial and bulk-like regions.

VIEs, pushing the mean value closer to that obtained in the isotropic simulation.

To account for this possibility, we performed much longer (1 ns) simulations for $\mathrm{I}^{-}(\mathrm{aq})$ and $\mathrm{SCN}^{-}(\mathrm{aq})$, so that we can carefully dissect the averaging based on the position of the ion relative to the GDS. In what follows, we partition the periodic slab simulation data into an "interfacial part" and a "bulk part", with the former defined to be those snapshots where the ion resides within a specified distance of the GDS. The VIE distribution can then be partitioned into interfacial and bulk contributions, and we will examine the sensitivity of this partition to the distance cutoff that is used to define the interfacial region.

Figure 3 presents the partitioned VIE distributions for both $\mathrm{I}^{-}(\mathrm{aq})$ and $\mathrm{SCN}^{-}(\mathrm{aq})$, obtained from 200 snapshots each separated in time by 5 ps. This is much better averaging than what is reported in Table 1, and is necessary in order to obtain good statistics for cases where the interfacial region is defined narrowly, e.g., GDS - $1 \AA$. We also consider partitions in which GDS $-3 \AA$ and also GDS $-5 \AA$ is used to separate the bulk and interfacial 
regions. Results from an isotropic simulation in a cubic unit cell serve as a fixed point of reference for bulk aqueous solution, and in every case the peak position from the isotropic simulation matches exactly with the bulk-like part of the periodic slab data. For $\mathrm{SCN}^{-}(\mathrm{aq})$, the peak of the VIE distribution is also exactly the same when the data are restricted to the interfacial region, no matter how narrowly defined, whereas for $\mathrm{I}^{-}(\mathrm{aq})$ the interfacial VIE peak is shifted to $0.2 \mathrm{eV}$ lower in energy in the interfacial region, for each of the three aforementioned definitions of the interfacial layer. That shift, however, is smaller than the width of either the bulk or the interfacial VIE distribution. We therefore conclude that interfacial VIE distributions are essentially indistinguishable from their bulk counterparts, for both $\mathrm{I}^{-}(\mathrm{aq})$ and $\mathrm{SCN}^{-}(\mathrm{aq})$.

C. Analysis of Short-Range Structure. We have documented that two solvation shells of explicit water molecules is enough to provide converged VIEs when appropriate boundary conditions are employed, and that VIEs computed for anions at the air/water interface differ from their bulk counterparts (if at all) by a shift that is comparable to (or smaller than) the inhomogeneous broadening of the VIE distribution. This implies that any shift in the VIE for $\mathrm{X}^{-}$(aq) between the bulk and interfacial environments is hardly distinguishable from thermal fluctuations. Taken together with the rapid convergence of the VIE with respect to explicit water, this would seem to suggest that the short-range ion-water hydrogen bonding around $\mathrm{X}^{-}(\mathrm{aq})$ might not be that different at the air/water interface as compared to the bulk aqueous environment. That hypothesis is explored in this section, by examining various structural parameters extracted from the MD simulations.

Figure 4 shows the time evolution of three quantities along $\mathrm{MD}$ trajectories for $\mathrm{I}^{-}(\mathrm{aq})$ and $\mathrm{SCN}^{-}(\mathrm{aq})$ : the distance between the ion and the GDS, $d_{\mathrm{GDS}}(t)$; the instantaneous number of ion-water hydrogen bonds, $n_{\mathrm{HB}}(t)$; and lastly $\operatorname{VIE}(t)$. (Criteria for counting hydrogen bonds are discussed in Section S1.4 and are consistent with several previous studies.) These simulations include about 600 ps of data where the ion is near the interface $\left(d_{\mathrm{GDS}}<5 \AA\right)$ along with an additional 400 ps where it moves farther away, into the bulk-like interior of the slab.

As the iodide ion moves to-and-fro relative to the GDS, which is defined by $d_{\mathrm{GDS}}=0$ in Fig. $4 \mathrm{a}$, fluctuations in $\operatorname{VIE}(t)$ appear to be completely uncorrelated with fluctuations in $d_{\mathrm{GDS}}(t)$. In particular, there is no discernible change in the VIE when the ion moves away from the GDS starting around $t=600$ ps. Fluctuations in $n_{\mathrm{HB}}(t)$ also appear to be largely uncorrelated, except that $\mathrm{I}^{-}(\mathrm{aq})$ in bulk water is characterized by an average of slightly less than one additional hydrogen bond, as compared to the interfacial structures. (For $\mathrm{I}^{-}$, similarities between bulk and interfacial solvation structure have been noted before, ${ }^{38,141}$ and these are further quantified below.) The apparent lack of correlation between $\operatorname{VIE}(t)$ and $n_{\mathrm{HB}}(t)$
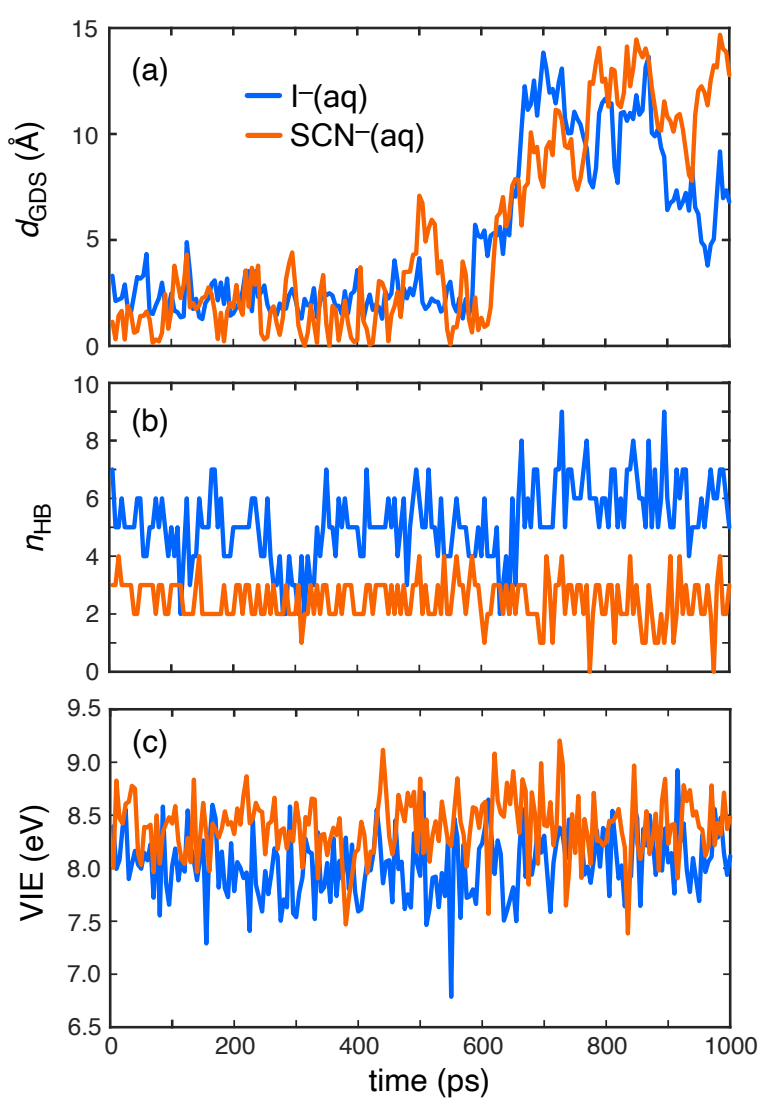

Fig. 4: Time evolution of various properties of $\mathrm{I}^{-}(\mathrm{aq})$ and $\mathrm{SCN}^{-}(\mathrm{aq})$ from periodic slab simulations: (a) the distance $\left(d_{\mathrm{GDS}}\right)$ between the ion and the GDS that defines the air/ water interface, (b) number of ion-water hydrogen bonds $\left(n_{\mathrm{HB}}\right)$, and (c) the VIE.

is surprising in view of the fact that each of the first few water molecules that is added around a gas-phase ion has a very significant impact on the VIE, as is clearly seen for $\mathrm{NO}_{3}^{-}(\mathrm{aq})$ in Fig. 1, yet transient fluctuations in the hydrogen-bonding network do not correlate with the VIE in any obvious way.

To further examine these correlations (or lack thereof), the simulation data from Fig. 4 are replotted as twodimensional scatter plots, comparing the distributions of $d_{\mathrm{GDS}}$ and the VIE (Fig. 5a), as well as $n_{\mathrm{HB}}$ versus VIE (Fig. 5b). In the former case, there is a cluster of data near $d_{\mathrm{GDS}}=0$, consistent with the surface propensity of $\mathrm{I}^{-}(\mathrm{aq})$, however the corresponding VIEs are spread over a range that is not dissimilar from the spread of the entire data set. This is consistent with the similarity between bulk and interfacial VIE distributions that was documented in Fig. 3. It is difficult to see any correlations at all in the $n_{\mathrm{HB}}$ versus VIE data. While transient fluctuations in the hydrogen-bond network are no doubt the origin of the inhomogeneous broadening of the VIE distribution, it is not so simple as saying that fewer hydrogen bonds to the ion implies a larger or smaller VIE, especially with a metric that discretizes the ion's hydra- 

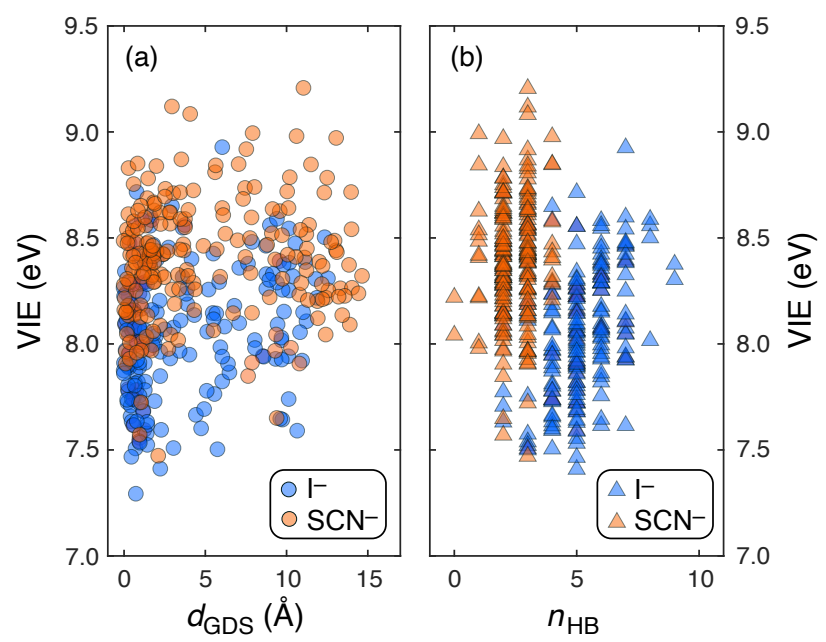

Fig. 5: Scatter plots of VIEs computed for $\mathrm{I}^{-}(\mathrm{aq})$ and $\mathrm{SCN}^{-}$(aq), versus (a) the distance from the ion to air/water interface, $d_{\mathrm{GDS}}$; and (b) the number of ion-water hydrogen bonds, $n_{\mathrm{HB}}$. These are the same data that are plotted as time series in Fig. 4.

tion shell into an integer number of hydrogen bonds.

In previous $\mathrm{MD}$ simulations of halide ions $\mathrm{A}^{-}(\mathrm{aq})$ at the air/water interface, Jungwirth and Tobias ${ }^{11}$ reported that the larger halides exhibit distinctive differences in terms of how their ion-water hydrogen bonds are oriented with respect to the surface normal, when compared to the same ions in bulk water. In that work, orientation with respect to the surface normal vector $\mathbf{r}_{\perp}$ was measured in terms of the angle it makes with $\mathbf{r}_{\mathrm{XO}}$, examining

$$
\cos \Theta_{\mathrm{AO} \perp}=\frac{\mathbf{r}_{\mathrm{AO}} \cdot \mathbf{r}_{\perp}}{\left\|\mathbf{r}_{\mathrm{AO}} \cdot \mathbf{r}_{\perp}\right\|} \text {. }
$$

We have analyzed our $\mathrm{I}^{-}(\mathrm{aq})$ and $\mathrm{SCN}^{-}(\mathrm{aq})$ simulations in the same way, examining the distribution of $\cos \left(\bar{\Theta}_{\mathrm{AO} \perp}\right)$, where $\bar{\Theta}_{\mathrm{AO} \perp}$ denotes the average value of $\Theta_{\mathrm{AO} \perp}$ considering each of the $n_{\mathrm{HB}}$ anion-oxygen vectors $\mathbf{r}_{\mathrm{AO}}$ around the ion. (For $\mathrm{SCN}^{-}$, we take $\mathrm{A}=\mathrm{N}$.) Distributions of $\cos \left(\bar{\Theta}_{\mathrm{AO} \perp}\right)$ are plotted in Fig. S9, separately for the the bulk and interfacial portions of the periodic slab data, with the latter partitioned using different definitions of the interfacial region, as in Fig. 3. Results for $\mathrm{I}^{-}(\mathrm{aq})$ are similar to what was reported in Ref. 11. For both $\mathrm{I}^{-}(\mathrm{aq})$ and $\mathrm{SCN}^{-}(\mathrm{aq})$, we find that $\mathbf{r}_{\mathrm{AO}}$ preferentially aligns towards the surface when the ion is in the interfacial region, whereas the distribution of angles is more isotropic when the ion resides in the interior of the slab. However, this alignment has seemingly no effect on the VIE. This is evident from Fig. S10, which presents a scatter plot of VIE versus $\cos \left(\bar{\Theta}_{\mathrm{AO} \perp}\right)$, analogous to the scatter plots in Fig. 5, and also from Fig. S11, which plots a joint probability distribution in VIE and $\cos \Theta_{\mathrm{AO} \perp}$, stripping away the averaging over first-shell hydrogen bonds.

Listed in Table 2 are the average structural parame- ters for $\mathrm{I}^{-}(\mathrm{aq}), \mathrm{SCN}^{-}(\mathrm{aq})$, and two other ions that exhibit surface propensity $\left(\mathrm{NO}_{3}^{-}\right.$and $\left.\mathrm{ClO}_{4}^{-}\right)$, along with one that does not $\left(\mathrm{NO}_{2}^{-}\right)$as a point of comparison. As in the analysis above, these data are taken from periodic slab simulations that are partitioned into bulk-like and interfacial regions, using GDS $-3 \AA$ to separate the two. In addition to $n_{\mathrm{HB}}$, the structural parameters examined in Table 2 include the average $\mathrm{A}^{-} \cdots \mathrm{H}$ hydrogen-bond distance $\left(\bar{r}_{\mathrm{HB}}\right)$ and average $\mathrm{H}-\mathrm{A}-\mathrm{O}\left(\bar{\theta}_{\mathrm{HB}}\right)$. Both quantities are averages over all $n_{\mathrm{HB}}$ ion-water hydrogen bonds in a given snapshot, and then the ensemble average affords the values reported as $\left\langle\bar{r}_{\mathrm{HB}}\right\rangle$ and $\left\langle\bar{\theta}_{\mathrm{HB}}\right\rangle$ in Table 2. Finally, $\left\langle\mathrm{CN}_{1}\right\rangle$ and $\left\langle\mathrm{CN}_{2}\right\rangle$ represent the average coordination numbers in the first and second solvation shells of the ion. The former is defined by the first local minimum in the $\mathrm{X} \cdots \mathrm{O}_{\mathrm{w}} \mathrm{RDF}$, where $\mathrm{X}$ is the central atom of the ion and $\mathrm{O}_{\mathrm{w}}$ denotes a water oxygen. (The RDFs that are used to make this determination are plotted in Fig. S3.) Due to the diffuse solvation structure around many of the soft ions, the value of $\mathrm{CN}_{2}$ is not defined by any RDF but is instead based on the number of water molecules contained within the cutoff distance that is needed to converge the VIE; see Section S1.4 for a discussion.

Note that $\left\langle\mathrm{CN}_{1}\right\rangle$ is considerably larger than $\left\langle n_{\mathrm{HB}}\right\rangle$ for each of the ions in Table 2, indicating that the first solvation shell defined by the RDF includes quite a few water molecules that are not directly hydrogen-bonded to the ion. This difference, $\left\langle\mathrm{CN}_{1}\right\rangle-\left\langle n_{\mathrm{HB}}\right\rangle$, ranges from 3.6 additional water molecules for $\mathrm{NO}_{3}^{-}$in bulk water (or 1.6 additional water molecules for $\mathrm{NO}_{3}^{-}$at the interface) up to 11.4 (bulk) and 7.5 (interface) in the case of $\mathrm{SCN}^{-}$. This may explain the lack of correlation between instantaneous values $n_{\mathrm{HB}}(t)$ and $\operatorname{VIE}(t)$ : even as hydrogen bonds to the ion are transiently broken and formed, the ion maintains a larger number of inner-sphere water molecules, polarization of which (upon ionization of the ion) may help to counterbalance fluctuations in the hydrogen-bonding environment.

In view of the significant difference between $\left\langle\mathrm{CN}_{1}\right\rangle$ and $\left\langle n_{\mathrm{HB}}\right\rangle$ when the ion resides in the bulk-like region of the slab, differences in $\left\langle\mathrm{CN}_{1}\right\rangle$ for the bulk versus interfacial environments seem less important. A reduction in $\left\langle\mathrm{CN}_{1}\right\rangle$ as the ion moves to the interface is expected, and arises at least partly (and perhaps mostly) from reduced water density at the interface. More significant, in our view, is the fact that the average number of ion-water hydrogen bonds is remarkably similar in both environments. The value of $\left\langle n_{\mathrm{HB}}\right\rangle$ at the interface does decrease by about one full hydrogen bond for $\mathrm{I}^{-}$and $\mathrm{NO}_{3}^{-}$, but is essentially unchanged for $\mathrm{ClO}_{4}^{-}$and $\mathrm{SCN}^{-}$. Values of $\bar{r}_{\mathrm{HB}}$ and $\bar{\theta}_{\mathrm{HB}}$ are essentially identical in both environments, for each of these ions. In particular, the ion-water hydrogen bonds are just as close to linearity $\left(\theta_{\mathrm{HB}}=0\right)$ at the air/water interface as they are in bulk water.

Compelling similarities between ion-water structural parameters in the bulk and interfacial environments come into better focus when presented in the form of radar 
Table 2: Average Structural Parameters for Aqueous Ions from Periodic Slab Simulations. ${ }^{a}$

\begin{tabular}{|c|c|c|c|c|c|c|c|c|c|c|}
\hline \multirow[t]{2}{*}{ Parameter } & \multicolumn{2}{|c|}{$\mathrm{NO}_{2}^{-}$} & \multicolumn{2}{|c|}{$\mathrm{NO}_{3}^{-}$} & \multicolumn{2}{|c|}{$\mathrm{ClO}_{4}^{-}$} & \multicolumn{2}{|c|}{$\mathrm{SCN}^{-}$} & \multicolumn{2}{|r|}{$\mathrm{I}^{-}$} \\
\hline & bulk & $\overline{\text { interface }}$ & bulk & $\overline{\text { interface }}$ & bulk & $\overline{\text { interface }}$ & bulk & $\overline{\text { interface }}$ & bulk & interface \\
\hline$\left\langle\mathrm{CN}_{1}\right\rangle^{b}$ & 11.4 & 8.9 & 9.0 & 8.0 & 14.0 & 11.0 & 14.0 & 10.0 & 11.0 & 7.0 \\
\hline$\left\langle\mathrm{CN}_{2}\right\rangle^{c}$ & 36.5 & 25.7 & 36.0 & 32.0 & 33.0 & 25.0 & 36.0 & 25.0 & 31.0 & 20.0 \\
\hline$\left\langle n_{\mathrm{HB}}\right\rangle^{d}$ & 5.0 & 4.8 & 5.4 & 4.4 & 6.5 & 6.6 & 2.6 & 2.5 & 6.0 & 5.2 \\
\hline$\left\langle\bar{r}_{\mathrm{HB}}\right\rangle^{e} / \AA$ & 1.9 & 1.9 & 1.9 & 1.9 & 2.2 & 2.2 & 2.1 & 2.1 & 2.9 & 2.8 \\
\hline$\left\langle\bar{\theta}_{\mathrm{HB}}\right\rangle^{f} / \mathrm{deg}$ & 6.9 & 6.7 & 7.6 & 7.0 & 8.5 & 7.5 & 7.2 & 6.8 & 6.8 & 5.8 \\
\hline
\end{tabular}

${ }^{a}$ Interfacial region is defined as GDS $-3 \AA .{ }^{b} \mathrm{CN}_{1}=$ number of first-shell water molecules, defined by the $\mathrm{X} \cdots \mathrm{O}_{\mathrm{w}}$ RDF. ${ }^{c} \mathrm{CN}_{2}=$ number of second-shell water molecules, defined by convergence of the VIE. ${ }^{d} n_{\mathrm{HB}}$ $=$ number of ion-water hydrogen bonds. ${ }^{e} \bar{r}_{\mathrm{HB}}$ is the average $\mathrm{A}^{-} \cdots \mathrm{H}$ distance for the $n_{\mathrm{HB}}$ hydrogen bonds around an instantaneous configuration of the ion, with $\mathrm{A}=\mathrm{O}$ for the oxyanions and $\mathrm{A}=\mathrm{N}$ for $\mathrm{SCN}^{-}$.

${ }^{f} \bar{\theta}_{\mathrm{HB}}$ is the average $\mathrm{H}-\mathrm{A}-\mathrm{O}$ angle for the $n_{\mathrm{HB}}$ hydrogen bonds around an instantaneous configuration of the ion. (A value $\theta_{\mathrm{HB}}=0$ indicates a linear hydrogen bond.)

(a) iodide

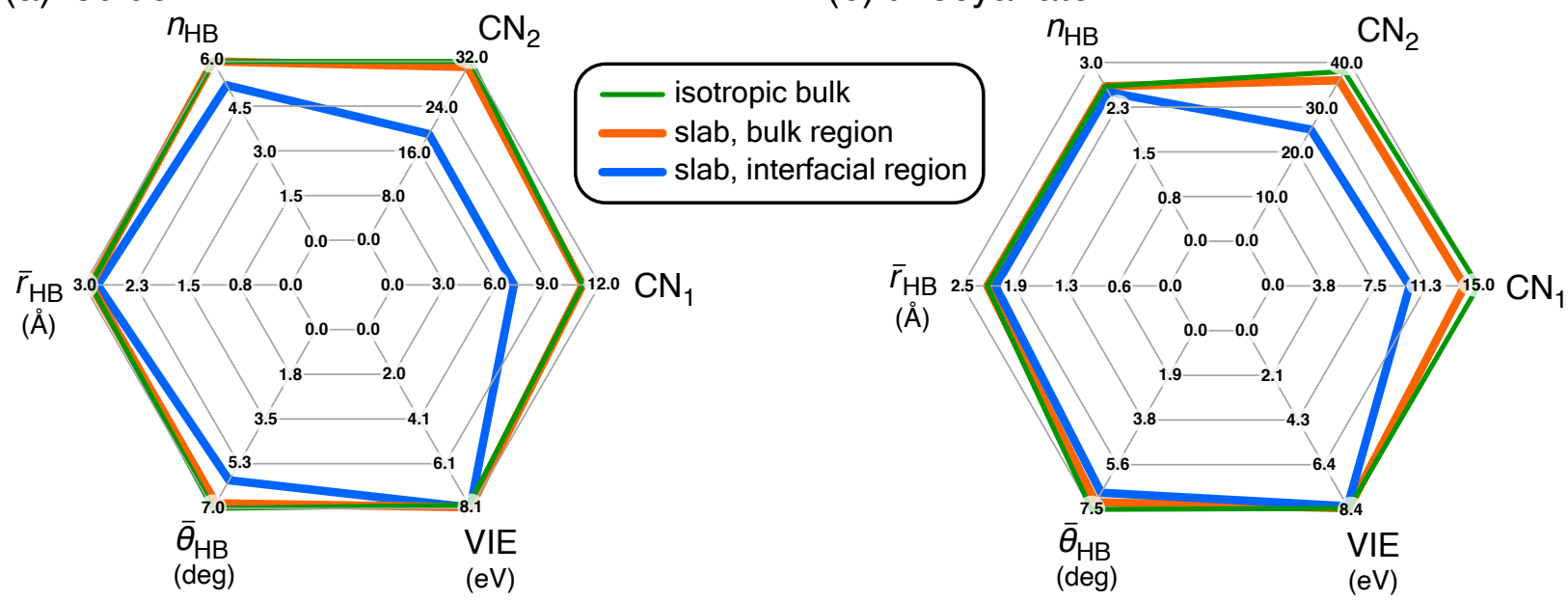

Fig. 6: Comparison of ensemble-averaged ion-water structural parameters for (a) $\mathrm{I}^{-}$(aq) and (b) $\mathrm{SCN}^{-}$(aq). Results are shown for both the bulk and interfacial regions of a periodic slab simulation (defined based on a GDS - $3 \AA$ partition), and also for an isotropic bulk simulation. Numerical data can be found in Table 2 and similar plots for $\mathrm{NO}_{3}^{-}(\mathrm{aq})$ and $\mathrm{ClO}_{4}^{-}(\mathrm{aq})$ can be found in Fig. S8.

plots; see Fig. 6 for $\mathrm{I}^{-}$and $\mathrm{SCN}^{-}$, and Fig. $\mathrm{S} 8$ for $\mathrm{NO}_{3}^{-}$ and $\mathrm{ClO}_{4}^{-}$. Apart from differences in the total first- and second-shell coordination numbers, which are manifestations of the reduced water density at the interface, the solvation structure is remarkably similar and this imparts a corresponding similarity upon the VIEs obtained from bulk versus interfacial regions of the water slab. The radar plots also compare averages obtained from bulklike interior region of the slab to those obtained from the isotropic bulk simulations that have no air/water interface. This comparison demonstrate that our preferred partition of the slab (GDS $-3 \AA$ ) affords a genuinely bulk-like region, where average structure parameters are indistinguishable from those computed in the isotropic simulations.

Structural parameters $\bar{r}_{\mathrm{HB}}$ and $\bar{\theta}_{\mathrm{HB}}$ represent averages over all $n_{\mathrm{HB}}$ ion-water hydrogen bonds in a given snapshot, but Fig. 7 strips away this averaging by plotting two-dimensional probability distributions in $\left(r_{\mathrm{HB}}, \theta_{\mathrm{HB}}\right)$, for both $\mathrm{I}^{-}(\mathrm{aq})$ and $\mathrm{SCN}^{-}$(aq), separately for the bulk and interfacial regions of each simulation. The distributions cluster around $r_{\mathrm{HB}}=2.5-2.8 \AA$ (iodide) and $r_{\mathrm{HB}}=1.9-2.2 \AA$ (thiocyanate) with $\theta_{\mathrm{HB}}$ centered around $3-10^{\circ}$ in either case, indicating nearly linear hydrogen bonds. Quasi-linear hydrogen bonding is driven by ionwater charge transfer, ${ }^{148}$ and in the case of $\mathrm{SCN}^{-}$it explains the smaller value of $\left\langle n_{\mathrm{HB}}\right\rangle$ as compared to the other ions (which is consistent with experiment), ${ }^{147}$ since it would be difficult to accommodate a larger value of near-linear hydrogen bonds around the linear $\mathrm{SCN}^{-}$moiety.

Apart from a slightly longer tail in the distribution of $\theta_{\mathrm{HB}}$, there is very little difference (for either ion) between the joint probability distribution $\left(r_{\mathrm{HB}}, \theta_{\mathrm{HB}}\right)$ that is obtained in the interior of the slab versus that obtained at the air/water interface. This is consistent with the similarity between bulk and interfacial VIEs, despite the fact that the latter property is sensitive to the short-range 

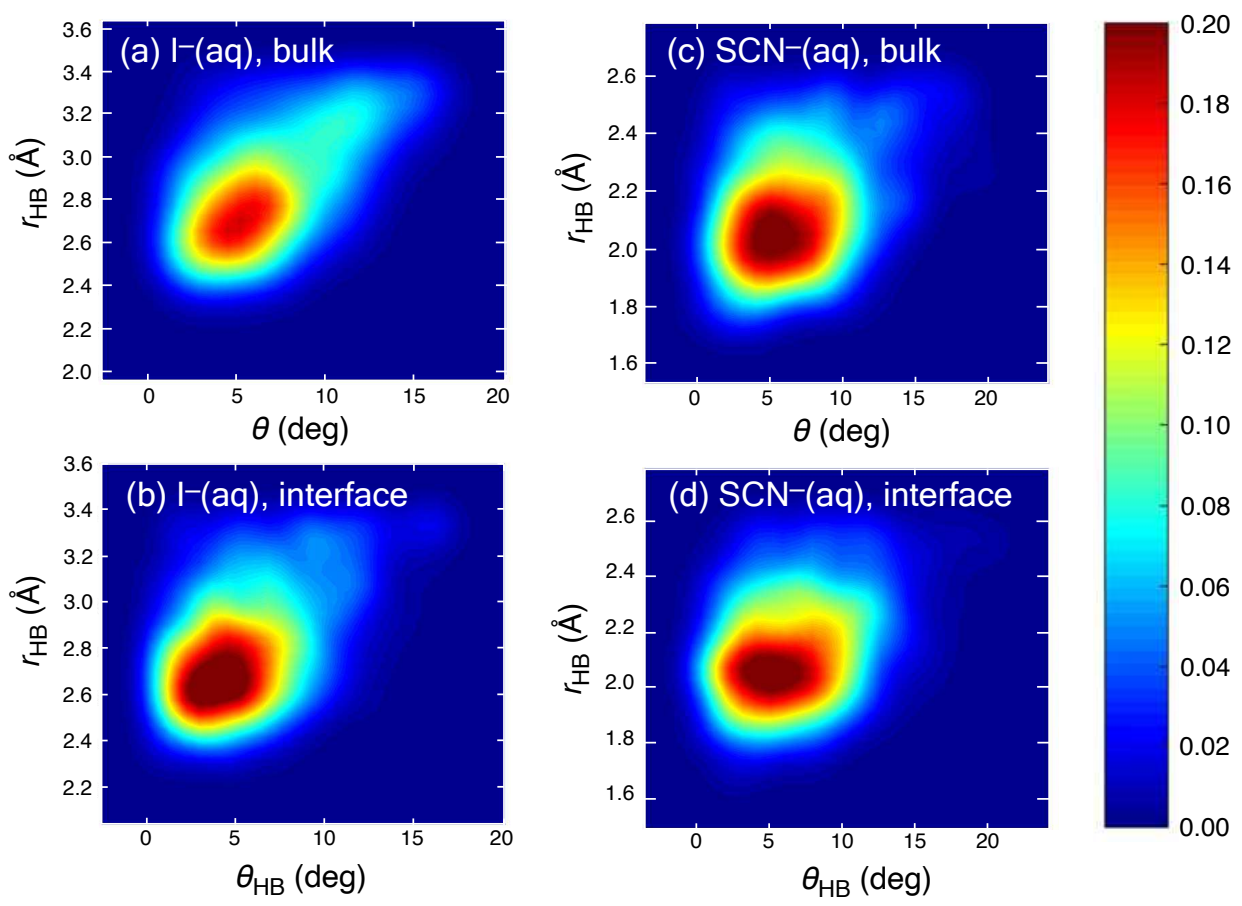

Fig. 7: Joint probability distributions in $\left(r_{\mathrm{HB}}, \theta_{\mathrm{HB}}\right)$ that characterize the hydrogen bond environment around $\mathrm{I}^{-}(\mathrm{aq})$ and $\mathrm{SCN}^{-}$(aq) in periodic slab simulations: (a) $\mathrm{I}^{-}$in the bulk (interior) region of the slab, (b) $\mathrm{I}^{-}$in the interfacial region, (c) $\mathrm{SCN}^{-}$in the bulk region, and (d) $\mathrm{SCN}^{-}$in the interfacial region. Unlike the data in Table 2 and Fig. 6, which are averages over all of the ion-water hydrogen bonds in a given snapshot (affording values denoted $\bar{r}_{\mathrm{HB}}$ and $\bar{\theta}_{\mathrm{HB}}$ ), these two-dimensional histograms include all of the ion-water hydrogen bonds, without averaging. The partition between bulk and interfacial regions of the periodic slab is defined by the criterion GDS $-3 \AA$.

hydrogen bonding evironment, as indicated by our convergence tests (Fig. 1). The present results support the idea that local solvation structure is hardly affected by the presence of the interface, even for surface-active ions such as $\mathrm{I}^{-}, \mathrm{SCN}^{-}, \mathrm{NO}_{3}^{-}$, and $\mathrm{ClO}_{4}^{-}$. Local solvation structure around these four anions spans a range from $\left\langle n_{\mathrm{HB}}\right\rangle=2.6$ for $\mathrm{SCN}^{-}$(aq) to $\left\langle n_{\mathrm{HB}}\right\rangle=6.5$ for $\mathrm{ClO}_{4}^{-}(\mathrm{aq})$, suggesting some generality to the conclusion that shortrange solvation structure of small inorganic ions is not much affected by the presence of the air/water interface.

One might hypothesize that the lack of any pronounced difference in the short-range hydration structure around an interfacial ion, relative to that in bulk water, arises because the putative "interfacial" ion is in reality enveloped by a layer of water even when $d_{\mathrm{GDS}}$ is small. This hypothesis is readily dismissed upon examination of the MD trajectories, which reveal that both $\mathrm{I}^{-}$and $\mathrm{SCN}^{-}$at the air/water interface spend a significant amount of time in "air-exposed" configurations; see Fig. S6. Other studies have also concluded that interfacial iodide ions reside primarily in the topmost layer of the solvent, ${ }^{38}$ and that the presence of various Hofmeister ions disrupts the water network only in the first solvation shell. ${ }^{21,118,149,150}$ The role of ion-induced water-water correlations in explaining ionic strength effects on water's surface tension has been specifically highlighted. ${ }^{151,152}$

An explanation for the structural similarities that is consistent with the air-exposed solvation motifs that we observe is that hydration of soft anions involves strong hydrogen bonds on only one side of the ion, even in bulk water, with a hydrophobic pocket emerging on the opposite side. This idea has been put forward previously with regard to $\mathrm{I}^{-}(\mathrm{aq}),{ }^{141} \mathrm{IO}_{3}^{-}(\mathrm{aq}),{ }^{144}$ and $\mathrm{SCN}^{-}(\mathrm{aq}),{ }^{144}$ and in particular the structural anisotropy around $\mathrm{I}^{-}(\mathrm{aq})$ that is reported in Ref. 141 is reproduced by the simulations reported here; see Fig. S12. As such, the airexposed configurations of the interfacial ion are not solvated very differently as compared to the same ion in bulk water. Other studies have also pointed to asymmetric solvation environments around anions (more so than cations), even in bulk water. ${ }^{153-156}$ Our results suggest that the solvation environments of various soft anions are asymmetric both in bulk water and at the air/water interface, leading to VIEs that are essentially indistinguishable.

\section{Conclusions}

The present work investigates ionization energies of inorganic anions in the aqueous phase, with particular focus on similarities between hydration motifs in bulk water versus hydration at the air/water interface. Liquid microjet photoelectron spectroscopy, which can measure 
the VIEs of these species in water, is mostly performed at photon energies where the attenuation length of the outgoing photoelectron is $1-10 \mathrm{~nm},{ }^{59,63}$ suggesting that the experiments are more sensitive to solutes at the interface. However, there has been little prior investigation of whether the VIEs themselves are sensitive to interfacial solvation, which is an especially relevant question for soft anions that are known to partition preferentially at the air/water interface. Our calculations suggest that differences between bulk and interfacial VIEs are inconsequential, which we attribute to similarities in first-shell hydration structure in both environments. These striking similarities serve as a reminder that although the structure of the air/water interface may be modified by the presence of dissolved ions, the hydration shell around a given solute may or may not be different than what it is in bulk aqueous solution. Whereas liquid microjet photoelectron spectroscopy may be surface-sensitive, our results strongly suggest that the observable that it measures is not surface-sensitive.

This work also reports a new computational protocol for aqueous VIEs. The existing conventional wisdom is that a prohibitively large number of explicit water molecules is needed to obtain converged VIEs in aqueous solution, ${ }^{87-92}$ but with an appropriate choice of continuum boundary conditions we find that convergence is achieved with two solvation shells. VIEs reported here are within $0.2-0.3 \mathrm{eV}$ of experimental values, even for polyvalent anions. This is comparable to the accuracy of the best existing periodic DFT calculations of the same quantities, ${ }^{86}$ but without the complexities posed by the periodic treatment when the number of electrons changes. ${ }^{128-135}$ The protocol reported here is also readily extensible to wave function-based quantum chemistry.

Rapid convergence of the VIE in our cluster-continuum protocol suggests that this quantity is mostly sensitive to short-range solvation structure, with the continuum boundary conditions making a large but non-specific contribution at longer range. Similarities in bulk and interfacial VIEs result from similarities in the short-range hydration environment. Our results are therefore consistent with the presumed surface-sensitivity of liquid microjet photoelectron spectroscopy, but also reveal that these values can likely be taken seriously as VIEs for bulk aqueous solutes. The latter are important parameters for the solution-phase theory of electron transfer. ${ }^{157-159}$

Observed similarities in the solvation structure of bulk versus interfacial anions sets up another interesting conundrum, namely, how to reconcile these observations with shifts in the $\mathrm{O}-\mathrm{H}$ stretching band of water are detected (using surface-selective vibrational spectroscopy) when soft ions are present at the air/water interface. ${ }^{22,23,49-55}$ Our results suggest that this is likely a second-shell (or possibly third-shell) solvation effect, resulting from subtle changes in the network of waterwater hydrogen bonds that are induced by the presence of the ion, rather than resulting directly from changes in ion-water hydrogen bonds at the interface. The surface sensitivity of soft anions is itself known to be a rather subtle effect, difficult to ascribe to changes in any single energetic term in the interaction potential, ${ }^{36,38}$ and at least partly driven by entropic considerations. ${ }^{32,38}$ The present work supports the idea that surface activity is likely a result of ion-induced changes in the structure of water itself, ${ }^{55}$ adding an aspect of nonlocal specificity to Hofmeister specific-ion effects.

\section{Acknowledgements}

This work was supported by National Science Foundation grant nos. CHE-1665322 and CHE-1955282. Calculations were performed at the Ohio Supercomputer Center under project no. PAA0003. ${ }^{160}$ We thank Steve Bradforth for providing an electronic copy of experimental data from Ref. 86 and Kevin Carter-Fenk for comments on the manuscript.

\section{Supporting Information Available}

Additional supporting calculations and analysis.

\section{References}

1 Jungwirth, P.; Tobias, D. J. Specific ion effects at the air/water interface. Chem. Rev. 2006, 106, 1259-1281.

2 Jungwirth, P.; Cremer, P. S. Beyond Hofmeister. Nature Chem. 2014, 6, 261-263.

3 Cacace, M. G.; Landau, E. M.; Ramsden, J. J. The Hofmeister series: Salt and solvent effects on interfacial phenomena. Q. Rev. Biophys. 1997, 30, 241-277.

4 Record, Jr., T. M.; Zhang, W.; Anderson, C. F. Analysis of effects of salts and uncharged solutes on protein and nucleic acid equilibria and processes; a practical guide to recognizing and interpreting polyelectrolyte effects, Hofmeister effects, and osmotic effects of salts. Adv. Protein Chem. 1998, 51, 281-353.

5 Zhang, Y.; Cremer, P. S. Chemistry of Hofmeister anions and osmolytes. Annu. Rev. Phys. Chem. 2010, 61, 63-83.

${ }^{6}$ Nostro, P. L.; Ninham, B. W. Hofmeister phenomena: An update on ion specificity in biology. Chem. Rev. 2012, 112, 2286-2322.

7 Jungwirth, P.; Tobias, D. J. Ions at the air/water interface. J. Phys. Chem. B 2002, 106, 6361-6373.

${ }^{8}$ Garrett, B. Ions at the air/water interface. Science 2004, 303, 1146-1147.

9 Jungwirth, P.; Winter, B. Ions at aqueous interfaces: From water surface to hydrated proteins. Annu. Rev. Phys. Chem. 2008, 59, 343-366.

10 Seinfeld, J. H. Meeting at the interface. Science 2000, 288, 285.

11 Jungwirth, P.; Tobias, D. J. Molecular structure of salt solutions: A new view of the interface with implications for heterogeneous atmospheric chemistry. J. Phys. Chem. B 2001, 105, 10468-10472. 
12 Tobias, D. J.; Stern, A. C.; Baer, M. D.; Levin, Y.; Mundy, C. J. Simulation and theory of ions at atmospherically relevant aqueous liquid-air interfaces. Annu. Rev. Phys. Chem. 2013, 64, 339-359.

13 Zhong, J.; Kumar, M.; Anglada, J. M.; MartinsCosta, M. T. C.; Ruiz-Lopez, M. F.; Zheng, X. C.; Francisco, J. S. Atmospheric spectroscopy and photochemistry at environmental interfaces. Annu. Rev. Phys. Chem. 2019, 70, 45-69.

14 Finlayson-Pitts, B. J.; Hemminger, J. C. Physical chemistry of airborne sea salt particles and their components. J. Phys. Chem. A 2000, 104, 11463-11477.

15 Knipping, E. M.; Lakin, M. J.; Foster, K. L.; Jungwirth, P.; Tobias, D. J.; Gerber, R. B.; Dabdub, D.; Finlayson-Pitts, B. J. Experiments and simulations of ionenhanced interfacial chemistry on aqueous $\mathrm{NaCl}$ aerosols. Science 2000, 288, 301-306.

16 Laskin, A.; Gaspar, D. J.; Wang, W.; Hunt, S. W.; Cowin, J. P.; Colson, S. D.; Finlayson-Pitts, B. J. Reactions at interfaces as a source of sulfate formation in sea-salt particles. Science 2003, 301, 340-344.

17 Finlayson-Pitts, B. J. The tropospheric chemistry of sea salt: A molecular-level view of the chemistry of $\mathrm{NaCl}$ and NaBr. Chem. Rev. 2003, 103, 4801-4822.

18 Gerber, R. B.; Varner, M. E.; Hammerich, A. D.; Riikonen, S.; Murdachaew, G.; Shemesh, D.; FinlaysonPitts, B. J. Computational studies of atmosphericallyrelevant chemical reactions in water clusters and on liquid water and ice surfacess. Acc. Chem. Res. 2015, 48, 399406.

19 Aagaard, K.; Carmack, E. C. The role of sea ice and other fresh water in the Arctic circulation. J. Geophys. Res. 1989, 94, 14485-14498.

20 Shcherbina, A. Y.; Talley, L. D.; Rudnick, D. L. Direct observation of North Pacific ventilation: Brine rejection in the Okhotsk Sea. Science 2003, 302, 1952-1955.

21 Song, J.; Kang, T. H.; Kim, M. W.; Han, S. Ion specific effects: Decoupling ion-ion and ion-water interactions. Phys. Chem. Chem. Phys. 2015, 17, 8306-8322.

${ }^{22}$ Liu, D.; Ma, G.; Levering, L. M.; Allen, H. C. Vibrational spectroscopy of aqueous sodium halide solutions and air-liquid interfaces: Observation of increased interfacial depth. J. Phys. Chem. B 2004, 108, 2252-2260.

23 Mucha, M.; Frigato, T.; Levering, L. M.; Allen, H. C.; Tobias, D. J.; Dang, L. X.; Jungwirth, P. Unified molecular picture of the surfaces of aqueous acid, base, and salt solutions. J. Phys. Chem. B 2005, 109, 7617-7623.

24 Petersen, P. B.; Saykally, R. J. Confirmation of enhanced anion concentration at the liquid water surface. Chem. Phys. Lett. 2004, 397, 51-55.

25 Petersen, P. B.; Saykally, R. J.; Mucha, M.; Jungwirth, P. Enhanced concentration of polarizable anions at the liquid water surface: SHG spectroscopy and MD simulations of sodium thiocyanide. J. Phys. Chem. B 2005, 109, 10915-10921. Erratum: J. Phys. Chem. B 2005, 109, 13402.

26 Petersen, P. B.; Saykally, R. J. Evidence for an enhanced hydronium concentration at the liquid water surface. J. Phys. Chem. B 2005, 109, 7976-7980.

27 Petersen, P. B.; Saykally, R. J. On the nature of ions at the liquid water surface. Annu. Rev. Phys. Chem. 2006, 57, 333-364.

28 Ghosal, S.; Hemminger, J. C.; Bluhm, H.; Mun, B. S.; Hebenstreit, E. L. D.; Ketteler, G.; Olgetree, D. F.;
Requejo, F. G.; Salmeron, M. Electron spectroscopy of aqueous solution interfaces reveals surface enhancement of halides. Science 2005, 307, 563-566.

29 Otten, D. E.; Shaffer, P. R.; Geissler, P. L.; Saykally, R. J. Elucidating the mechanism of selective ion adsorption to the liquid water surface. Proc. Natl. Acad. Sci. USA 2012, 109, 701-705. Erratum: Proc. Natl. Acad. Sci. USA 2012, 109, 3190.

30 Petersen, M. K.; Iyengar, S. S.; Day, T. J. F.; Voth, G. A. The hydrated proton at the water liquid/vapor interface. J. Phys. Chem. B 2004, 108, 14803-14806.

31 Dang, L. X. Computational study of ion binding to the liquid interface of water. J. Phys. Chem. B 2002, 106, 10388-10394.

32 Herce, D. H.; Perera, L.; Darden, T. A.; Sagui, C. Surface solvation for an ion in a water cluster. J. Chem. Phys. 2005, 122, 024513.

33 Archontis, G.; Leontidis, E.; Andreou, G. Attraction of iodide ions by the free water surface, revealed by simulations with a polarizable force field based on Drude oscillators. J. Phys. Chem. B 2005, 109, 17957-17966.

34 Chang, T. M.; Dang, L. X. Recent advances in molecular simulations of ion solvation at liquid interfaces. Chem. Rev. 2006, 106, 1305-1322.

35 Ishiyama, T.; Morita, A. Molecular dynamics study of gas-liquid aqueous sodium halide interfaces. I. Flexible and polarizable molecular modeling and interfacial properties. J. Phys. Chem. C 2007, 111, 721-737.

36 Caleman, C.; Hub, J. S.; van Maaren, P. J.; van der Spoel, D. Atomistic simulation of ion solvation in water explains surface preference of halides. Proc. Natl. Acad. Sci. USA 2011, 108, 6838-6842.

37 Dweik, J.; Srour, M.; Karaky, K.; Kobeissi, M.; Joumaa, W.; Abou-Saleh, K. Molecular simulation of ion transport at the water/vapor interface. Open J. Phys. Chem. 2012, 2, 147-155.

38 Stern, A. C.; Baer, M. D.; Mundy, C. J.; Tobias, D. J. Thermodynamics of iodide adsorption at the instantaneous air-water interface. J. Chem. Phys. 2013, 138, 114709.

39 Sun, L.; Li, X.; Tu, Y.; Ågren, H. Origin of ion selectivity at the air/water interface. Phys. Chem. Chem. Phys. 2015, 17, 4311-4318.

40 Pearson, R. G. Hard and soft acids and bases, HSAB, part I: Fundamental principles. J. Chem. Educ. 1968, 45, 581-587.

41 Pearson, R. G. Hard and soft acids and bases, HSAB, part II: Underlying theories. J. Chem. Educ. 1968, 45, 643-648.

42 Pearson, R. G. Chemical hardness and density functional theory. J. Chem. Sci. 2005, 117, 369-377.

43 Ayers, P. W. The physical basis of the hard/soft acid/base principle. Faraday Discuss. 2007, 135, 161-190.

44 Jørgensen, C. K. Electric polarizability, innocent ligands and spectroscopic oxidation states. Struct. Bond. 1966, 1, 234-248.

45 Jungwirth, P.; Tobias, D. J. Chloride anions on aqueous clusters, at the air-water interface, and in liquid water: Solvent effects on $\mathrm{Cl}^{-}$polarizability. J. Phys. Chem. A 2002, 106, 379-383.

46 Jungwirth, P.; Curtis, J. E.; Tobias, D. J. Polarizability and aqueous solvation of the sulfate dianion. Chem. Phys. Lett. 2003, 367, 704-710.

47 Salvador, P.; Curtis, J. E.; Tobias, D. J.; Jungwirth, P. 
Polarizability of the nitrate anion and its solvation at the air/water interface. Phys. Chem. Chem. Phys. 2003, 5, 3752-3757.

$48 \mathrm{Ab}$ initio calculations suggest that the polarizability of $\mathrm{SO}_{4}^{2-}(\mathrm{aq})$ is about $7 \AA^{3},{ }^{46}$ whereas for $\mathrm{Cl}^{-}(\mathrm{aq})$ it is $4 \AA^{3},{ }^{45}$ and for $\mathrm{NO}_{3}^{-}(\mathrm{aq})$ the isotropic polarizability is $4.9 \AA^{3} .{ }^{47}$ Nevertheless, $\mathrm{SO}_{4}^{2-}(\mathrm{aq})$ exhibits no surface propensity whatsoever, while the two less-polarizable ions do partition preferentially at the air/water interface.

49 Verreault, D.; Hua, W.; Allen, H. C. From conventional to phase-sensitive vibrational sum frequency generation spectroscopy: Probing water organization at aqueous interfaces. J. Phys. Chem. Lett. 2012, 3, 3012-3028.

50 Raymond, E. A.; Richmond, G. L. Probing the molecular structure and bonding of the surface of aqueous salt solutions. J. Phys. Chem. B 2004, 108, 5051-5059.

51 Tarbuck, T. L.; Ota, S. T.; Richmond, G. L. Spectroscopic studies of solvated hydrogen and hydroxide ions at aqueous surfaces. J. Am. Chem. Soc. 2006, 128, 1451914527.

52 Levering, L. M.; Sierra-Hernández, R.; Allen, H. C. Observation of hydronium ions at the air-aqueous acid interface: Vibrational spectroscopic studies of aqueous $\mathrm{HCl}$, HBr, and HI. J. Phys. Chem. C 2007, 111, 8814-8826.

53 Viswanath, P.; Aroti, A.; Motschmann, H.; Leontidis, E. Vibrational sum frequency generation spectroscopic investigation of the interaction of thiocyanate ions with zwitterionic phospholipid monolayers at the air-water interface. J. Phys. Chem. B 2009, 113, 14816-14823.

54 Jubb, A. M.; Hua, W.; Allen, H. C. Environmental chemistry at vapor/water interfaces: Insights from vibrational sum frequency generation spectroscopy. Annu. Rev. Phys. Chem. 2012, 63, 107-130.

55 Roy, S.; Mondal, J. A. "Breaking" and "making" of water structure at the air/water-electrolyte $\left(\mathrm{NaXO}_{3} ; \mathrm{X}=\mathrm{Cl}\right.$, Br, I) interface. J. Phys. Chem. Lett. 2021, 12, 19551960.

56 Winter, B.; Faubel, M. Photoemission from liquid aqueous solutions. Chem. Rev. 2006, 106, 1176-1211.

57 Seidel, R.; Thürmer, S.; Winter, B. Photoelectron spectroscopy meets aqueous solution: Studies from a vacuum liquid microjet. J. Phys. Chem. Lett. 2011, 2, 633-641.

58 Faubel, M.; Siefermann, K. R.; Liu, Y.; Abel, B. Ultrafast soft X-ray photoelectron spectroscopy at liquid water microjets. Acc. Chem. Res. 2012, 45, 120-130.

59 Seidel, R.; Winter, B.; Bradforth, S. E. Valence electronic structure of aqueous solutions: Insights from photoelectron spectroscopy. Annu. Rev. Phys. Chem. 2016, 67, 283-305.

60 Suzuki, T. Ultrafast photoelectron spectroscopy of aqueous solutions. J. Chem. Phys. 2019, 151, 090901.

61 Dupuy, R.; Richter, C.; Winter, B.; Meijer, G.; Schlögl, R.; Bluhm, H. Core level photoelectron spectroscopy of heterogeneous reactions at liquid-vapor interfaces: Current status, challenges, and prospects. J. Chem. Phys. 2021, 154, 060901.

62 Jablonski, A.; Powell, C. J. Relationships between electron inelastic mean free paths, effective attenuation lengths, and mean escape depths. J. Electron Spectrosc. 1999, 100, 137-160.

63 Ottoson, N.; Faubel, M.; Bradforth, S. E.; Jungwirth, P.; Winter, B. Photoelectron spectroscopy of liquid water and aqueous solution: Electron effective attenuation lengths and emission-angle anisotropy. J. Electron
Spectrosc. 2010, 177, 60-70.

64 Suzuki, Y.-I.; Nishizawa, K.; Kurahashi, N.; Suzuki, T. Effective attenuation length of an electron in liquid water between 10 and $600 \mathrm{eV}$. Phys. Rev. E 2014, 90, 010302R.

65 Yamamoto, Y.; Karashima, S.; Adachi, S.; Suzuki, T. Wavelength dependence of UV photoemission from solvated electrons in bulk water, methanol, and ethanol. J. Phys. Chem. A 2016, 120, 1153-1159.

66 Luckhaus, D.; Yamamoto, Y.; Suzuki, T.; Signorell, R. Genuine binding energy of the hydrated electron. Sci. Adv. 2017, 3, e1603224.

67 Nishitani, J.; Yamamoto, Y.; West, C. W.; Karashima, S.; Suzuki, T. Binding energy of solvated electrons and retrieval of true UV photoelectron spectra of liquids. Sci. Adv. 2019, 5, eaaw6896.

68 Signorell, R. Can current experimental data exclude nonGaussian genuine band shapes in ultraviolet photoelectron spectra of the hydrated electron? J. Phys. Chem. Lett. 2020, 11, 1516-1519.

69 Malerz, S. et al. Low-energy constraints on photoelectron spectra measured from liquid water and aqueous solutions. Phys. Chem. Chem. Phys. 2021, .

${ }^{70}$ Kurahashi, N.; Karashima, S.; Tang, Y.; Horio, T.; Abulimiti, B.; Suzuki, Y.-I.; Ogi, Y.; Oura, M.; Suzuki, T. Photoelectron spectroscopy of aqueous solutions: Streaming potentials of $\mathrm{NaX}(\mathrm{X}=\mathrm{Cl}, \mathrm{Br}$, and $\mathrm{I})$ solutions and electron binding energies of liquid water and $\mathrm{X}^{-}$. J. Chem. Phys. 2014, 140, 174506.

71 Olivieri, G.; Goel, A.; Kleibert, A.; Cvetko, D.; Brown, M. A. Quantitative ionization energies and work functions of aqueous solutions. Phys. Chem. Chem. Phys. 2016, 18, 29506-29515.

72 Nishitani, J.; Karashima, S.; West, C. W.; Suzuki, T. Surface potential of liquid microjet investigated using extreme ultraviolet photoelectron spectroscopy. J. Chem. Phys. 2020, 152, 144503.

73 Olivieri, G.; Parry, K. M.; Powell, C. J.; Tobias, D. J.; Brown, M. A. Quantitative interpretation of molecular dynamics simulations for x-ray photoelectron spectroscopy of aqueous solutions. J. Chem. Phys. 2016, 144, 154704.

74 Kusaka, R.; Nihonyanagi, S.; Tahara, T. The photochemical reaction of phenol becomes ultrafast at the air-water interface. Nat. Chem. 2021,

75 Rizzuto, A. M.; Irgen-Gioro, S.; Eftekhari-Bafrooei, A.; Saykally, R. J. Broadband deep UV spectra of interfacial aqueous iodide. J. Phys. Chem. Lett. 2016, 7, 3882-3885.

76 Mizuno, H.; Rizzuto, A. M.; Saykally, R. J. Chargetransfer-to-solvent spectrum of thiocyanate at the air/ water interface measured by broadband deep ultraviolet electronic sum frequency generation spectroscopy. J. Phys. Chem. Lett. 2018, 9, 4753-4757.

77 Sagar, D. M.; Bain, C. D.; Verlet, J. R. R. Hydrated electrons at the water/air interface. J. Am. Chem. Soc. 2010, 132, 6917-6919.

78 Buchner, F.; Schultz, T.; Lübcke, A. Solvated electrons at the water-air interface: Surface versus bulk signal in low kinetic energy photoelectron spectroscopy. Phys. Chem. Chem. Phys. 2012, 14, 5837-5842.

79 Matsuzaki, K.; Kusaka, R.; Nihonyanagi, S.; Yamaguchi, S.; Nagata, T.; Tahara, T. Partially hydrated electrons at the air/water interface observed by UV-excited time-resolved heterodyne-detected vibrational sum frequency generation spectroscopy. J. Am. Chem. Soc. 2016, 138, 7551-7557. 
80 Coons, M. P.; You, Z.-Q.; Herbert, J. M. The hydrated electron at the surface of neat liquid water appears to be indistinguishable from the bulk species. J. Am. Chem. Soc. 2016, 138, 10879-10886.

81 Coons, M. P.; Herbert, J. M. Quantum chemistry in arbitrary dielectric environments: Theory and implementation of nonequilibrium Poisson boundary conditions and application to compute vertical ionization energies at the air/water interface. J. Chem. Phys. 2018, 148, 222834. Erratum: J. Chem. Phys. 2019, 151, 189901.

82 Herbert, J. M.; Coons, M. P. The hydrated electron. Annu. Rev. Phys. Chem. 2017, 68, 447-472.

83 Herbert, J. M. Structure of the aqueous electron. Phys. Chem. Chem. Phys. 2019, 21, 20538-20565.

${ }^{84}$ Pohl, M. N.; Muchová, E.; Seidel, R.; Ali, H.; Š. Sršeň,; Wilkinson, I.; Winter, B.; Slavíček, P. Do water's electrons care about electrolytes? Chem. Sci. 2019, 10, 848865.

85 Weber, R.; Winter, B.; Schmidt, P. M.; Widdra, W.; Hertel, I. V.; Dittmar, M.; Faubel, M. Photoemission from aqueous alkali-metal-iodide salt solutions using EUV synchrotron radiation. J. Phys. Chem. A 2004, 108, 4729-4736.

86 Pham, T. A.; Govoni, M.; Seidel, R.; Bradforth, S. E.; Schwegler, E.; Galli, G. Electronic structure of aqueous solutions: Bridging the gap between theory and experiments. Sci. Adv. 2017, 3, e1603210.

87 Jacobson, L. D.; Herbert, J. M. A one-electron model for the aqueous electron that includes many-body electronwater polarization: Bulk equilibrium structure, vertical electron binding energy, and optical absorption spectrum. J. Chem. Phys. 2010, 133, 154506.

${ }^{88}$ Ghosh, D.; Isayev, O.; Slipchenko, L. V.; Krylov, A. I. Effect of solvation on the vertical ionization energy of thymine: From microhydration to bulk. J. Phys. Chem. A 2011, 115, 6028-6038.

89 Ghosh, D.; Roy, A.; Seidel, R.; Winter, B.; Bradforth, S.; Krylov, A. I. First-principle protocol for calculating ionization energies and redox potentials of solvated molecules and ions: Theory and application to aqueous phenol and phenolate. J. Phys. Chem. B 2012, 116, 72697280 .

90 Cauët, E.; Valiev, M.; Weare, J. H. Vertical ionization potentials of nucleobases in a fully solvated DNA environment. J. Phys. Chem. B 2010, 114, 5886-5894.

91 Tazhigulov, R. N.; Gurunathan, P. K.; Kim, Y.; Slipchenko, L. V.; Bravaya, K. B. Polarizable embedding for simulating redox potentials of biomolecules. Phys. Chem. Chem. Phys. 2019, 21, 11642-11650.

92 Tóth, Z.; Kubečka, J.; Muchová, E.; Slaviček, P. Ionization energies in solution with QM:QM approach. Phys. Chem. Chem. Phys. 2020, 22, 10550-10560.

93 Mukherjee, M.; Tripathi, D.; Brehm, M.; Riplinger, C.; Dutta, A. K. Efficient EOM-CC-bassed protocol for the calculation of electron affinity of solvated nucleobases: Uracil as a case study. J. Chem. Theory Comput. 2021, 17, 105-116.

94 D'Annibale, V.; Nardi, A. N.; Amadei, A.; D'Abramo, M. Theoretical characterization of the reduction potentials of nucleic acids in solution. J. Chem. Theory Comput. 2021, 17, 1301-1307.

${ }^{95}$ Herbert, J. M. Dielectric continuum methods for quantum chemistry. WIREs Comput. Mol. Sci. 2021, e1519.

96 Ren, P.; Ponder, J. W. Polarizable atomic multipole wa- ter model for molecular mechanics simulation. J. Phys. Chem. B 2003, 107, 5933-5947.

97 Ren, P.; Ponder, J. W. Temperature and pressure dependence of the AMOEBA water model. J. Phys. Chem. B 2004, 108, 13427-13437.

98 Ponder, J. W.; Wu, C.; Ren, P.; Pande, V. S.; Chodera, J. D.; Schnieders, M. J.; Haque, I.; Mobley, D. L.; Lambrecht, D. S.; R. A. DiStasio, J.; HeadGordon, M.; Clark, G. N. I.; Johnson, M. E.; HeadGordon, T. Current status of the AMOEBA polarizable force field. J. Phys. Chem. B 2010, 114, 2549-2564.

99 Grossfield, A.; Ren, P.; Ponder, J. W. Ion solvation thermodynamics from simulation with a polarizable force field. J. Am. Chem. Soc. 2003, 125, 15671-15682.

100 Jiao, D.; King, C.; Grossfield, A.; Darden, T. A.; Ren, P. Simulation of $\mathrm{Ca}^{2+}$ and $\mathrm{Mg}^{2+}$ solvation using polarizable atomic multipole potential. J. Phys. Chem. B 2006, 110, 18553-18559.

101 Lambrecht, D. S.; Clark, G. N. I.; Head-Gordon, T.; Head-Gordon, M. Exploring the rich energy landscape of sulfate-water clusters $\mathrm{SO}_{4}^{2-}\left(\mathrm{H}_{2} \mathrm{O}\right)_{n=3-7}$ : An electronic structure approach. J. Phys. Chem. A 2011, 115, 1143811454.

102 Ren, P.; Wu, C.; Ponder, J. W. Polarizable atomic multipole-based molecular mechanics for organic molecules. J. Chem. Theory Comput. 2011, 7, 3143-3161.

103 Lagardère, L.; Jolly, L.-H.; Lipparini, F.; Aviat, F.; Stamm, B.; Jing, Z. F.; Harger, M.; Torabifard, H.; Cisneros, G. A.; Schneiders, M. J.; Gresh, N.; Maday, Y.; Ren, P. Y.; Ponder, J. W.; Piquemal, J.-P. Tinker-HP: A massively parallel molecular dynamics package for multiscale simulations of large complex systems with advanced point dipole polarizable force fields. Chem. Sci. 2018, 9, 956-972.

104 Pliego Jr., J. R.; Riveros, J. M. Hybrid discretecontinuum solvation methods. WIREs Comput. Mol. Sci. 2020, 10, e1440.

105 Stein, C. J.; Herbert, J. M.; Head-Gordon, M. The Poisson-Boltzmann model for implicit solvation of electrolyte solutions: Quantum chemical implementation and assessment via Sechenov coefficients. J. Chem. Phys. 2019, 151, 224111.

106 Lange, A. W.; Herbert, J. M.; Albrecht, B. J.; You, Z.-Q. Intrinsically smooth discretization of Connolly's solvent-excluded molecular surface. Mol. Phys. 2020, 118, e1644384.

107 Mardirossian, N.; Head-Gordon, M. $\omega B 97 M-V$ : A combinatorially optimized, range-separated hybrid, metaGGA density functional with VV10 nonlocal correlation. J. Chem. Phys. 2016, 144, 214110.

108 Sherrill, C. D.; Lee, M. S.; Head-Gordon, M. On the performance of density functional theory for symmetrybreaking problems. Chem. Phys. Lett. 1999, 302, 425430.

109 Wille, U.; Dreessen, T. Mechanistic insights into $\mathrm{NO}_{3}^{\bullet}$ induced self-terminating radical oxygenations, part 1: A computational study on $\mathrm{NO}_{3}^{\bullet}$ and its addition to alkynes. J. Phys. Chem. A 2006, 110, 2195-2203.

110 Dasgupta, S.; Herbert, J. M. Standard grids for highprecision integration of modern density functionals: SG-2 and SG-3. J. Comput. Chem. 2017, 38, 869-882.

111 Shao, Y. et al. Advances in molecular quantum chemistry contained in the Q-Chem 4 program package. Mol. Phys. 2015, 113, 184-215. 
112 Winter, B.; Weber, R.; Widdra, W.; Dittman, M.; Faubel, M.; Hertel, I. V. Full valence band photoemission from liquid water using EUV synchrotron radiation. J. Phys. Chem. A 2004, 108, 2625-2632.

113 Perry, C. F.; Zhang, P.; Nunes, F. B.; Jordan, I.; von Conta, A.; Wörner, H. J. Ionization energy of liquid water revisted. J. Phys. Chem. Lett. 2020, 11, 1789-1794.

114 Perry, C. F.; Jordan, I.; Zhang, P.; von Conta, A.; Nunes, F. B.; Wörner, H. J. Photoelectron spectroscopy of liquid water with tunable extreme-ultraviolet radiation: Effects of electron scattering. J. Phys. Chem. Lett. 2021, 12, 2990-2996.

115 Gilbert, A. T. B.; Besley, N. A.; Gill, P. M. W. Selfconsistent field calculations of excited states using the maximum overlap method (MOM). J. Phys. Chem. A 2008, 112, 13164-13171.

116 Barca, G. M. J.; Gilbert, A. T. B.; Gill, P. M. W. Simple models for difficult electronic excitations. J. Chem. Theory Comput. 2018, 14, 1501-1509.

117 Carter-Fenk, K.; Herbert, J. M. State-targeted energy projection: A simple and robust approach to orbital relaxation of non-aufbau self-consistent field solutions. J. Chem. Theory Comput. 2020, 16, 5067-5082.

118 Collins, K. D.; Neilson, G. W.; Enderby, J. E. Ions in water: Characterizing the forces that control chemical processes and biological structure. Biophys. Chem. 2007, 128, 95-104.

119 Kiriukhin, M. Y.; Collins, K. D. Dynamic hydration numbers for biologically important ions. Biophys. Chem. 2002, 99, 155-168.

120 Lange, A. W.; Herbert, J. M. A smooth, nonsingular, and faithful discretization scheme for polarizable continuum models: The switching/Gaussian approach. J. Chem. Phys. 2010, 133, 244111.

121 You, Z.-Q.; Mewes, J.-M.; Dreuw, A.; Herbert, J. M. Comparison of the Marcus and Pekar partitions in the context of non-equilibrium, polarizable-continuum reaction-field solvation models. J. Chem. Phys. 2015, 143, 204107.

122 Mewes, J.-M.; You, Z.-Q.; Wormit, M.; Kriesche, T.; Herbert, J. M.; Dreuw, A. Experimental benchmark data and systematic evaluation of two a posteriori, polarizablecontinuum corrections for vertical excitation energies in solution. J. Phys. Chem. A 2015, 119, 5446-5464.

123 Mewes, J.-M.; Herbert, J. M.; Dreuw, A. On the accuracy of the state-specific polarizable continuum model for the description of correlated ground and excited states in solution. Phys. Chem. Chem. Phys. 2017, 19, 1644-1654.

${ }^{124}$ Hiranuma, Y.; Kaniwa, K.; Shoji, M.; Mafuné, F. Solvation structures of iodide on and below a surface of aqueous solution studied by photodetachment spectroscopy. J. Phys. Chem. A 2011, 115, 8493-8497.

125 West, C. W.; Nishitani, J.; Higashimura, C.; Suzuki, T. Extreme ultraviolet time-resolved photoelectron spectroscopy of aqueous aniline solution: Enhanced surface concentration and pump-induced space charge effect. Mol. Phys. 2020, 119, e1748240.

126 Leslie, M.; Gillan, M. J. The energy and elastic dipole tensor of defects in ionic crystals calculated by the supercell method. J. Phys. C: Solid State 1985, 18, 973-982.

127 Schultz, P. A. Local electrostatic moments and periodic boundary conditions. Phys. Rev. B 1999, 60, 1551-1554.

128 Schultz, P. A. Theory of defect levels and the "band gap problem" in silicon. Phys. Rev. Lett. 2006, 96, 246401.
129 Alkauskas, A.; Broqvist, P.; Pasquarello, A. Defect energy levels in density functional calculations: Alignment and band gap problem. Phys. Rev. Lett. 2008, 101, 046405 .

130 Costanzo, F.; Sulpizi, M.; Della Valle, R. G.; Sprik, M. The oxidation of tyrosine and tryptophan studied by a molecular dynamics normal hydrogen electrode. J. Chem. Phys. 2011, 134, 244508.

131 Cheng, J.; Sprik, M. Alignment of electronic energy levels at electrochemical interfaces. Phys. Chem. Chem. Phys. 2012, 14, 11245-11267.

132 Opalka, D.; Pham, T. A.; Sprik, M.; Galli, G. Electronic energy levels and band alignment for aqueous phenol and phenolate from first principles. J. Phys. Chem. B 2015, 119, 9651-9660.

133 Ambrosio, F.; Miceli, G.; Pasquarello, A. Redox levels in aqueous solution: Effect of van der Waals interactions and hybrid functionals. J. Chem. Phys. 2015, 143, 244508.

134 Ambrosio, F.; Guo, Z.; Pasquarello, A. Absolute energy levels of liquid water. J. Phys. Chem. Lett. 2018, 9, 32123216.

135 Tölle, J.; Gomes, A. S. P.; Ramos, P.; Pavanello, M. Charged-cell periodic DFT simulations via an impurity model based on density embedding: Application to the ionization potential of liquid water. Int. J. Quantum Chem. 2019, 119, e25801.

136 Koneshan, S.; Rasaiah, J. C.; Lynden-Bell, R. M.; Lee, S. H. Solvent structure, dynamics, and ion mobility in aqueous solutions at $25^{\circ} \mathrm{C}$. J. Phys. Chem. B 1998, 102, 4193-4204.

137 Kropman, M. F.; Bakker, H. J. Dynamics of water molecules in aqueous solvation shells. Science 2001, 291, 2118-2120.

138 Heuft, J. M.; Meijer, E. J. Density functional theory based molecular-dynamics study of aqueous iodide solvation. J. Chem. Phys. 2005, 123, 094506.

139 Viswanath, P.; Motschmann, H. Oriented thiocyanate anions at the air-electrolyte interface and its implications on interfacial water-a vibrational sum frequency spectroscopy study. J. Phys. Chem. C 2007, 111, 4484-4486.

140 Viswanath, P.; Motschmann, M. Effect of interfacial presence of oriented thiocyanate on water structure. J. Phys. Chem. C 2008, 112, 2099-2103.

141 Wick, C. D.; Xantheas, S. S. Computational investigation of the first solvation shell structure of interfacial and bulk aqueous chloride and iodide ions. J. Phys. Chem. B 2009, 113, 4141-4146.

142 Pham, V. T.; Tavernelli, I.; Milne, C. J.; van der Veen, R. M.; D'Angelo, P.; Bressler, C.; Chergui, M. The solvent shell structure of aqueous iodide: X-ray absorption spectroscopy and classical, hybrid QM/MM and full quantum molecular dynamics simulations. 2010, 371, 24-29.

143 Maruani, J.; Kuleff, A. I.; Chong, D. P.; Bonnelle, C. Ansatz for the evaluation of the relativistic contributions to core ionization energies in complex molecules involving heavy atoms. Int. J. Quantum Chem. 2005, 104, 397-410.

144 Baer, M. D.; Mundy, C. J. An ab initio approach to understanding the specific ion effect. Faraday Discuss. 2013, 160, 89-101.

145 Onorato, R. M.; Otten, D. E.; Saykally, R. J. Adsorption of thicyanate ions to the dodecanol/water interface characterized by UV second harmonic generation. Proc. Natl. Acad. Sci. USA 2009, 106, 15176-15180. 
146 Riès-Kautt, M.; Ducruix, A. Inferences drawn from physicochemical studies of crystallogenesis and precrystalline state. In Macromolecular Crystallography Part A, Vol. 276; Carter, Jr., C. W., Ed.; Academic Press: 1997; Chapter 3, pages 23-59.

147 Mason, P. E.; Neilson, G. W.; Dempsey, C. E.; Barnes, A. C.; Cruickshank, J. M. The hydration structure of guanidinium and thiocyanate ions: Implications for protein stability iin aqueous solution. Proc. Natl. Acad. Sci. USA 2003, 100, 4557-4561.

148 Herbert, J. M.; Carter-Fenk, K. Electrostatics, charge transfer, and the nature of the halide-water hydrogen bond. J. Phys. Chem. A 2021, 125, 1243-1256.

149 Smith, J. D.; Saykally, R. J.; Geissler, P. L. The effects of dissolved halide anions on hydrogen bonding in liquid water. J. Am. Chem. Soc. 2007, 129, 13847-13856.

150 Mancinelli, R.; Botti, A.; Bruni, F.; Ricci, M. A.; Soper, A. K. Hydration of sodium, potassium, and chloride ions in solution and the concept of structure maker/ breaker. J. Phys. Chem. B 2007, 111, 13570-13577.

151 Chen, Y.; Okur, H. I.; Gomopoulos, N.; MaciasRomero, C.; Cremer, P. S.; Petersen, P. B.; Tocci, G.; Wilkins, D. M.; Liang, C.; Ceriotti, M.; Roke, S. Electrolytes induce long-range orientational order and free energy changes in the H-bond network of bulk water. Sci. Adv. 2016, 2, e1501891.

152 Okur, H. I.; Chen, Y.; Wilkins, D. M.; Roke, S. The Jones-Ray effect reinterpreted: Surface tension minima of low ionic strength electrolyte solutions are caused by elec- tric field induced water-water correlations. Chem. Phys. Lett. 2017, 684, 433-442.

153 Carignano, M. A.; Karlström, G.; Linse, P. Polarizable ions in polarizable water: A molecular dynamics study. J. Phys. Chem. B 1997, 101, 1142-1147.

154 Garde, S.; Hummer, G.; Paulaitis, M. E. Free energy of hydration of a molecular ionic solute: Tetramethylammonium ion. J. Chem. Phys. 1998, 108, 1552-1561.

155 Raugel, S.; Klein, M. L. An ab initio study of water molecules in the bromide ion solvation shell. J. Chem. Phys. 2002, 116, 196-202.

156 Rajamani, S.; Ghosh, T.; Garde, S. Size dependent ion hydration, its asymmetry, and convergence to macroscopic hydration. J. Chem. Phys. 2004, 120, 4457-4466.

157 Warshel, A. Dynamics of reaction in polar solvents. Semiclassical trajectory studies of electron-transfer and proton-transfer reactions. J. Phys. Chem. 1982, 86, 22182224.

158 King, G.; Warshel, A. Investigation of the free energy functions for electron transfer reactions. J. Chem. Phys. 1990, 93, 8682-8692.

159 VandeVondele, J.; Ayala, R.; Sulpizi, M.; Sprik, M. Redox free energies and one-electron energy levels in density functional theory based ab initio molecular dynamics. J. Electroanal. Chem. 2007, 607, 113-120.

160 "Ohio Supercomputer Center", http://osc.edu/ark:/19495/f5s1ph73. 
TABLE OF CONTENTS GRAPHIC

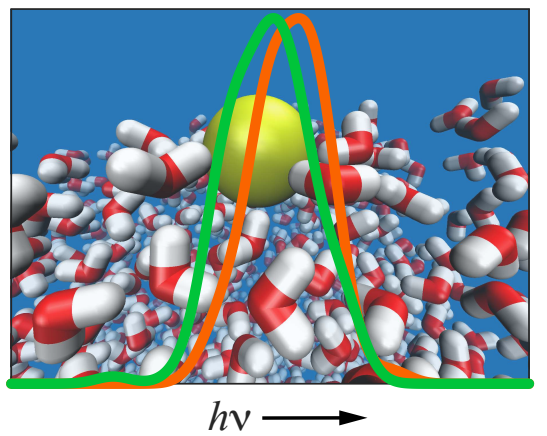

